\title{
Patterned, organoid-based cartilaginous implants exhibit zone specific functionality forming osteochondral-like tissues in vivo
}

Gabriella Nilsson Hall ${ }^{1,2}$, Wai Long Tam², Konstantinos S. Andrikopoulos ${ }^{3,4}$, Leire CasasFraile $^{5}$, George A. Voyiatzis ${ }^{3}$, Liesbet Geris ${ }^{1,6,7}$, Frank P. Luyten ${ }^{1,2, \text { * }^{*}, \text { Ioannis Papantoniou }}{ }^{1,}$ $2,3,+* *$

${ }^{1}$ Prometheus Division of Skeletal Tissue Engineering, KU Leuven, O\&N1, Herestraat 49, PB 813, 3000 Leuven, Belgium

${ }^{2}$ Skeletal Biology and Engineering Research Center, Department of Development and Regeneration, KU Leuven, O\&N1, Herestraat 49, PB 813, 3000 Leuven, Belgium

${ }^{3}$ Institute of Chemical Engineering Sciences, Foundation for Research and Technology-Hellas, Stadiou 26504, Platani, Patras, Greece

${ }^{4}$ Department of Physics, University of Patras, GR-265 00 Rio-Patras, Greece

${ }^{5}$ Laboratory of Tissue Homeostasis and Disease, Skeletal Biology and Engineering Research Center, Department of Development and Regeneration, KU Leuven, O\&N1, Herestraat 49, PB 813, Leuven 3000, Belgium

${ }^{6}$ GIGA in silico medicine, Université de Liège, Avenue de l'Hôpital 11 - BAT 34, 4000 Liège 1, Belgium

${ }^{7}$ Biomechanics Section, KU Leuven, Celestijnenlaan 300C, PB 2419, 3001 Leuven, Belgium

$\dagger$ Co-senior authors.

* Corresponding Authors: Skeletal Biology and Engineering Research Center; Onderwijs en Navorsing 8th floor, bus 813; B-3000 Leuven, Belgium

Tel: +3216346138

Fax: +32 16346200

Email: ioannis.papantoniou@kuleuven.be

Email: frank.luyten@kuleuven.be 


\begin{abstract}
Tissue engineered constructs have the potential to respond to the unmet medical need of treating deep osteochondral defects. However, current tissue engineering strategies struggle in the attempt to create patterned constructs with biologically distinct functionality. In this work, a developmentally-inspired modular approach is proposed, whereby distinct cartilaginous organoids are used as living building blocks. First, a hierarchical construct was created, composed of three layers of cartilaginous tissue intermediates derived from human periosteumderived cells: (i) early (SOX9), (ii) mature (COL2) and (iii) (pre)hypertrophic (IHH, COLX) phenotype. Subcutaneous implantation in nude mice generated a hybrid tissue containing one mineralized and one non-mineralized part. However, the non-mineralized part was represented by a collagen type I positive fibrocartilage-like tissue. To engineer a more stable articular cartilage part, iPSC-derived cartilage microtissues (SOX9, COL2; IHH neg) were generated. Subcutaneous implantation of assembled iPSC-derived cartilage microtissues resulted in a homogenous cartilaginous tissue positive for collagen type II but negative for osteocalcin. Finally, iPSC-derived cartilage microtissues in combination with the pre-hypertrophic cartilage organoids (IHH, COLX) could form dual tissues consisting of i) a cartilaginous safranin $\mathrm{O}$ positive and ii) a bony osteocalcin positive region upon subcutaneous implantation, corresponding to the pre-engineered zonal pattern. The assembly of functional building blocks, as presented in this work, opens possibilities for the production of complex tissue engineered implants by embedding zone-specific functionality through the use of pre-programmed living building blocks.
\end{abstract}




\section{Graphical Abstract}

Chondrogenic Differentiation

Organoid Self-assembly into Zonated Implant

in-vivo Implantation

Zone-specific Functionality
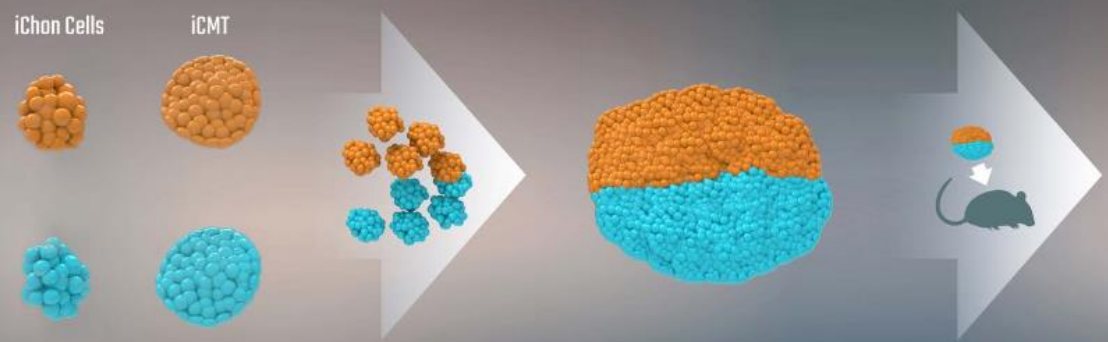

Cartiloge

hPOC Cells $\mathrm{Imm}$

Keywords: tissue engineering; osteochondral; induced pluripotent stem cells; organoid; patterned implant 


\section{Introduction}

An osteochondral defect is a local injury of the joint affecting the articular cartilage and the underlying subchondral bone [1]. Deep osteochondral defects caused by trauma have a high prevalence in young patients and can result in substantial functional disability, pain, swelling and are a high risk factor for the onset of osteoarthritis when left untreated [2-4]. Osteoarthritis (OA) is a joint disease affecting the whole joint but in particular the osteochondral unit [5]. To prevent the onset of posttraumatic OA in otherwise healthy joints, treatment of deep osteochondral defects $(>8 \mathrm{~mm})$ requires implants that represent the full osteochondral unit of articular cartilage and subchondral bone [6]. In the future, osteochondral implants may also be an option for treatment in more advanced OA. However, current treatment methods are suboptimal and associated with donor-site morbidity and/or limited tissue availability [7]. Novel advancements in tissue engineering (TE) could provide future solutions for efficient and synchronized regeneration of the articular cartilage and subchondral bone, irrespective of the size of the defect. However, current TE strategies entail drawbacks with regard to the formation of patterned osteochondral-like tissues.

To date, TE constructs developed for creation of osteochondral tissues have applied scaffolds created from hydrogels [8], polymers in combination with ceramics [9] and native cartilage extracellular matrix (ECM) [10] either alone or seeded with chondrocytes and/or adult progenitor cell types $[11,12]$. A majority of efforts employed single cell populations which, upon implantation as large implants, have shown impaired cell viability and regenerative potential [13]. In addition, the large volumetric presence of foreign material has recently been shown to affect the regenerative processes in an in vivo setting [14]. At the same time, the use of certain calcium phosphate $(\mathrm{CaP})$ have been shown to trigger immune reactions from the host with detrimental effects on the implanted cells [15]. Moreover, many of these TE constructs are composed of two considerably different zones either in terms of biology (for example using 
terminally differentiated cells), or in terms of material (using high volumes of polymers and $\mathrm{CaP})$ which have very different properties from the native tissue $[3,6]$.

Recent advances in the field of TE demonstrated that in vitro recapitulation of processes during skeletal development can lead to the development of functional intermediate tissues that upon implantation in vivo mature into full organs [16-20]. The long bones with edges covered by articular cartilage develop via a cartilage intermediate, where mesenchymal condensation is followed by chondrogenic maturation creating a cartilage template. The chondrogenic differentiation follows a distinct cellular cascade from proliferating chondrocytes (Sox9), prehypertrophic chondrocytes (Sox9, Foxa2) to hypertrophic chondrocytes (Runx2, Sp7) which promote tissue mineralization and remodeling into bone while articular cartilage is formed at the template edges by Gdf5 positive progenitor cells [21-24]. Regarding osteochondral tissues, a distinct engineered cartilage intermediate in vitro could potentially mature into nonmineralized, mineralized cartilage or bone upon implantation in vivo. Cartilage intermediate tissues from lapine bone marrow-derived mesenchymal stromal cells (BMSC) or human periosteum derived cells (hPDC) have been demonstrated to contribute to the regeneration of osteochondral defects, illustrating the power of developmentally inspired approaches [25,26]. However, the cartilage quality was suboptimal and the constructs failed to capture the zonal nature of the native joint containing several zones being articular cartilage, mineralized cartilage and subchondral bone. In addition, subchondral bone originates from the cartilage template while cells in the articular cartilage are also derived from invading $G d f 5$ positive cells, which may make it challenging to create osteochondral grafts in vitro using a single cell type under the same culture conditions [27,28]. Efforts have been made to allow gradient maturation within one tissue by applying different media from the top to the bottom of the constructs [29] but this makes the process complex and may limit the future possibility for upscaling.

Therefore, the zonal complexity of osteochondral tissues implicates that a bottom-up approach, where different building blocks are assembled into a multi-layered structure, is more 
attractive [30-32]. We recently reported a developmentally inspired bottom-up approach for successful healing of critical size long bone defects through assembly of prehypertrophic microtissues ("callus organoids") which upon implantation mimicked the function of the soft callus during fracture healing. Furthermore, gene signatures attained from transcriptomic analysis demonstrated that the differentiation into "callus organoids" followed the developmental process of endochondral ossification from a proliferative to a prehypertrophic stage [19]. Hence, the sequentially differentiated building blocks have potential for the formation of a hierarchical osteochondral tissue.

To generate cell-based constructs, a major challenge with the use of adult mesenchymal stromal cells (MSCs) is the formation of hyaline-like Saf O positive cartilage, rich in collagen type II. Instead, mostly fibrocartilage rich in collagen type I is formed and because of its suboptimal mechanical properties, clinical success is limited $[33,34]$. Articular chondrocytes (ACs) isolated from the articular surface have shown promising results for the healing of full thickness cartilage defects but isolation and extended expansion leads to dedifferentiation [35]. For these reasons, induced pluripotent stem cells (iPSCs) are emerging as a promising cell source for cartilage TE and hyaline-like Saf O positive cartilage tissue has successfully been produced from human iPSCs [36-38]. Additionally, the iPSC-derived engineered cartilage tissue demonstrated phenotypic stability in both rat and mini-pig cartilage defect models [37].

Hence, osteochondral TE constructs should be designed to allow synchronized formation of both articular cartilage and the underlying subchondral bone. In the current study, we applied a bottom-up strategy to create patterned constructs. In order to obtain best performance for both layers, distinct cartilaginous microtissue populations derived from hPDCs or iPSCs were developed to form a subchondral and articular cartilage zone, respectively. We hypothesized that cartilage microtissues (iCMTs) from iPSC-derived chondrocytes (iChon) in combination with bone forming "callus organoids" (COs) from hPDCs could be engineered into 
cartilaginous intermediate implants that possess zone specific characteristics resulting in bone and cartilage formation upon subcutaneous implantation.

\section{Materials and Methods}

\section{Periosteal cell isolation and culture}

hPDCs were isolated from periosteal biopsies of five female donors of age $14 \pm 3$ years old $(n=5)$ from which a single cell pool was created as previously described [39]. Cells were seeded at a density of 5700 cells $/ \mathrm{cm}^{2}$ for expansion until passage 9 (in vitro experiment) and 7 (in vivo experiments) at $37^{\circ} \mathrm{C}, 5 \% \mathrm{CO}_{2}$ and $95 \%$ humidity in Dulbecco's modified Eagle's medium (DMEM, Life Technologies, UK) with $10 \%$ fetal bovine serum (HyClone FBS, Thermo Scientific, USA), $1 \%$ antibiotic-antimycotic (100units/mL penicillin, 100mg/mL streptomycin and $0.25 \mathrm{mg} / \mathrm{mL}$ amphotericin B) and $1 \mathrm{mM}$ sodium pyruvate (Life Technologies, UK). Expansion medium was changed every 2-3 days and cells were harvested with TrypLE ${ }^{\mathrm{TM}}$ Express (Life Technologies, UK) at a confluence of 90\%. TrypLETM Express was used for all passaging and harvesting steps during cell handling. Patients' informed consent were obtained and all procedures were approved (ML7861) by the ethical committee for Human Medical Research (KU Leuven).

Formation of agarose microwells and cartilaginous microtissues from human periosteum derived cells

Polydimethylsiloxane (PDMS, Dow Corning Sylgard 184 elastomer, MAVOM Chemical Solutions) molds were fabricated as described elsewhere [40]. Briefly, an SU8 wafer was fabricated to produce a PDMS mold containing pillars with a diameter of $200 \mu \mathrm{m}$ and height of $153 \mu \mathrm{m}$. Next, 3\% agarose (Thermo Fisher) was carefully poured over the PDMS mold and let to cool down to form agarose microwells (153 $\mu \mathrm{m}$ depth, $200 \mu \mathrm{m}$ diameter). The agarose microwells were punched into a size $\left(1.8 \mathrm{~cm}^{2}\right)$ to fit in a 24 -well plate resulting in approximately 
2000 microwells per 24 well plate well. Agarose microwells were sterilized under UV before seeding 500000 hPDCs per well resulting in microspheroids with approximately 250 cells/spheroid. Microspheroids were cultured in xeno-free chemically defined chondrogenic medium (CM1) (LG-DMEM (Gibco) supplemented with $1 \%$ antibiotic-antimycotic (100units $/ \mathrm{mL}$ penicillin, $100 \mathrm{mg} / \mathrm{mL}$ streptomycin and $0.25 \mathrm{mg} / \mathrm{mL}$ amphotericin $\mathrm{B}$ ), $1 \mathrm{mM}$ ascorbate- 2 phosphate, $100 \mathrm{nM}$ dexamethasone, $40 \mu \mathrm{g} / \mathrm{mL}$ proline, $20 \mu \mathrm{M}$ of Rho-kinase inhibitor Y27632 (Axon Medchem), ITS+ Premix Universal Culture Supplement (Corning) (including $6.25 \mu \mathrm{g} / \mathrm{mL}$ insulin, $6.25 \mu \mathrm{g} / \mathrm{mL}$ transferrin, $6.25 \mu \mathrm{g} / \mathrm{mL}$ selenious acid, $1.25 \mu \mathrm{g} / \mathrm{mL}$ bovine serum albumin (BSA), and $5.35 \mu \mathrm{g} / \mathrm{mL}$ linoleic acid), $100 \mathrm{ng} / \mathrm{mL}$ BMP-2 (INDUCTOS ${ }^{\circledR}$ ), 100 ng/mL GDF5 (PeproTech), 10 ng/mL TGFß1 (PeproTech), 1 ng/mL BMP-6 (PeproTech) and $0.2 \mathrm{ng} / \mathrm{mL}$ FGF-2 (R\&D systems)) [41] for up to three weeks with media changes 2 times per week.

\section{Fluorescent staining for viability and proliferation}

Cell viability in microspheroids was assessed qualitatively with Live-or-Dye NucFix ${ }^{\text {TM }}$ Red Staining Kit (Biotium, US). Briefly, microspheroids were washed with Phosphate buffered saline (PBS) and Live-or-Dye NucFix ${ }^{\mathrm{TM}}$ Red Dye (1:1000 in PBS) was added for 30 minutes at $37^{\circ} \mathrm{C}, 5 \% \mathrm{CO}_{2}$ and $95 \%$ humidity followed by fixation with $4 \%$ paraformaldehyde (PFA) at room temperature, permeabilization and staining with 4',6-diamidino-2-phenylindole (DAPI) $(2.5 \mu \mathrm{g} / \mathrm{mL})$. Cell proliferation in microspheroids was visualized using the Click-iT® EdU (5ethynyl-2'-deoxyuridine) Imaging Kit (Life Technologies, USA) according to the manufacturer's protocol. Briefly, $10 \mu \mathrm{M}$ EdU was added to microspheroids during four days. Next, microsperoids were fixed in 4\% PFA, EdU was detected with Alexa Fluor azide and cells were counter stained with Hoechst $33342(5 \mu \mathrm{g} / \mathrm{mL})$. Stained microspheroids were mounted on glass slides using ProLong ${ }^{\mathrm{TM}}$ Glass Antifade Mountant (Invitrogen, US) and imaged with widefield fluorescence microscopy using Olympus IX83 inverted microscope equipped with DP73 
camera taking images each $5 \mu \mathrm{m}$ followed by merging. The percentage of EdU/Hoechst (proliferating/all cells) stained area was quantified using ImageJ software (NIH) [42] for 18-20 microspheroids per time point.

\section{Generation of iPSC derived chondrocytes for dual implants}

The human iPSC line CY2 (Rutgers University Cell and DNA Repository) was cultured as previously described [37]. In summary, hiPSCs were maintained on mitomycin-c (Sigma) treated SNL feeder cells in human embryonic stem cell medium (Dulbecco's modified eagle medium (DMEM) Ham's F12 nutrient mix (F12) (Sigma) supplemented with 20\% knockout serum replacement, $2 \mathrm{mM}$ Glutamax, $1 \%$ sodium pyruvate (SP), $1 \%$ non-essential amino acids (NEAA), $0.1 \mathrm{mM}$ 2-mercaptoethanol (2ME), $50 \mathrm{U}$ and $50 \mathrm{mg} / \mathrm{ml}$ penicillin/streptomycin (Pen/Strep) and $10 \mathrm{ng} / \mathrm{ml}$ human basic fibroblast growth factor (FGF-2, Peprotech)) at $37^{\circ} \mathrm{C}$ and $5 \% \mathrm{CO}_{2}$. Medium refreshment was performed daily and cells were passaged once a week onto fresh feeder cells. hiPSCs were transferred and maintained in feeder-free conditions using Essential 8 (Thermo Fisher) and Matrigel (Becton Dickinson) coated 6-well plates (Nunc ${ }^{\mathrm{TM}}$ Thermo Scientific ${ }^{\mathrm{TM}}$ ) followed by chondrogenic differentiation according to a previously described protocol [37]. Primitive streak and mesoderm induction were carried out for 72 hours by using $8 \mu \mathrm{M}$ canonical wingless related integration protein (Wnt) agonist CHIR99021 (GSK3 inhibitor, Axon Medchem), 8-20 ng/ml FGF-2 and $1 \mu \mathrm{M}$ retinoic acid (RA). Subsequently, chondrogenic differentiation was performed until day 14 using chondrogenic medium (CM2) (DMEM supplemented with 1\% Fetal Bovine Serum (FBS, Hyclone), 1\% L-Glutamine, 1\% NEAA, $1 \%$ SP, $1 \%$ Insulin-Transferrin-Selenite $X$ (ITS-X), $50 \mu \mathrm{g} / \mathrm{ml}$ ascorbic acid (AA, Sigma), 0.1 mM 2ME, 10 ng/ml FGF-2, 10 ng/ml TGF- $\beta 1,10$ ng/ml BMP2, 10 ng/ml GDF5 (Prospec), $50 \mathrm{U}$ and $50 \mathrm{mg} / \mathrm{ml}$ Pen/Strep). After 14 days, cartilage-like nodules were cultured in suspension using CM2 without FGF-2 for 8 weeks. Next, cartilage-like nodules were 
digested to release and isolate human iChon. The nodules were incubated with rotation for 4 hrs at $37^{\circ} \mathrm{C}$ and $5 \% \mathrm{CO}_{2}$ in digestion medium (DMEM: F12 (Gibco) supplemented with 1.5 mg/mL collagenase B (Roche), 1\% Antibiotics-Antimycotics: Gibco, 10\% FBS: Hyclone SV30160.03).

\section{Formation of hiPSC derived cartilage microtissues}

Agarose microwells were created using MicroTissues ${ }^{\circledR}$ 3D Petri Dish ${ }^{\circledR}$ micro-molds (Sigma) for 12 -well plates according to manufacturer's description. Briefly, $500 \mu \mathrm{L}$ of melted $2 \%$ agarose (Thermo Fisher) was pipetted into the precision micro-molds containing 256 pillars (800 $\mu \mathrm{m}$ depth, $400 \mu \mathrm{m}$ dimeter). After the agarose was gelled, it was removed and transferred to a 12 well and an additional of $500 \mu \mathrm{L}$ agarose (2\%) was added around the insert for immobilization. The microwells were sterilized under UV before use. iChon were resuspended in CM2 to a concentration of 128000 cell/ $100 \mu \mathrm{L}$ and $100 \mu \mathrm{L}$ was seeded into the microwell. Cells were let to sediment for 20 minutes after which $1 \mathrm{~mL}$ CM2 was added and cultured for 3 weeks at $37^{\circ} \mathrm{C}, 5 \% \mathrm{CO}_{2}$ and $95 \%$ to form iCMTs.

\section{Isolation and expansion of human articular chondrocytes as adult chondrocyte control}

Adult human articular chondrocytes (hAC) were used to compare the chondrogenic phenotype of iPSC derived chondrocytes. The hACs were obtained from patients undergoing hip replacement for osteoporotic or malignancy-associated fractures (female: 82, male: 54 and male 84 years old). The University Hospitals Leuven Ethics Committee and Biobank Committee approved the study. For chondrocytes isolation, cartilage was dissected from the joint explant surfaces and then rinsed with saline. The tissue was cut into small pieces, using a sterile surgical blade. Cartilage explants were incubated with $2 \mathrm{mg} / \mathrm{ml}$ pronase solution (Roche) for $90 \mathrm{~min}$ at $37^{\circ} \mathrm{C}$ under continuous agitation and digested overnight at $37^{\circ} \mathrm{C}$ in $1.5 \mathrm{mg} / \mathrm{ml}$ collagenase $\mathrm{B}$ solution (Roche). The preparation was filtered through a $70 \mu \mathrm{M}$ strainer and cells were plated in culture flasks and cultured in a humidified atmosphere at $37^{\circ} \mathrm{C}, 5 \% \mathrm{CO}_{2}$ with a density of 16 
000 cells $\mathrm{cm}^{-2}$. Culture medium consisted of DMEM/F12 (Gibco), 10\% fetal bovine serum (FBS) (Gibco), 1\% (vol/vol) antibiotic/antimycotic (Gibco) and 1\% L-glutamine (Gibco). Experiments were performed with passage 2 cells in triplicate.

\section{Dual building block fusion assay}

Microtissues were carefully flushed out from their microwells and seeded onto another microwell insert already containing microtissues. Wells containing two microtissues were imaged with 10x magnification over time in a well plate incubator (OKOlab Top Stage Incubator with insert H301-EC-24MW) using an inverted microscope (Olympus IX53) previously described in [43]. The length of the duplets and width of each microtissue was manually measured and the aspect ratio (AR) was defined as length divided by the average widths (Figure 3c).

\section{Formation of zonal constructs}

PDMS cylinders ( $2 \mathrm{~mm}$ diameter, $5 \mathrm{~mm}$ depth) were placed in a petri-dish and $3 \%$ agarose was carefully poured to cover the PDMS cylinders. The agarose was left to gel where after a $6 \mathrm{~mm}$ agarose part was punched out, the PDMS pillar was removed and the now created macro-well was placed in a ThinCert ${ }^{\mathrm{TM}}$ Cell Culture Inserts (Greiner Bio-One) for 24 well plates. The macro-wells were sterilized under UV before use. Next, three-layered hierarchical intermediate tissues with progressive stages of chondrogenic maturation were created layer-by-layer (Figure 1a). Day 7 and day 14 hPDC microtissues represented the cartilaginous part and day $21 \mathrm{hPDC}$ microtissues ("callus organoids") represented the hypertrophic part. For this, approximately 1000 building blocks of day 7 hPDC microtissues were carefully flushed out from their microwells. Building blocks were centrifuged and resuspended in $20 \mu \mathrm{L}$ fresh CM1 media followed by seeded into the macro-well $(2 \mathrm{~mm} \varnothing)$ to allow sedimentation and fusion at $37^{\circ} \mathrm{C}, 5 \% \mathrm{CO} 2$ and 95\% humidity during 1 hour. Subsequently, the second building block population (day 14 hPDC microtisues) was seeded on top of the underlying tissue followed by 1-hour fusion. The third and final building block population (day $21 \mathrm{hPDC}$ microtissues) was seeded and allowed to 
fuse for 1 hour followed by careful addition of CM1 media. The three-layered hierarchical intermediate tissues were ready for analysis after 24 hours of additional fusion. For the creation of dual constructs with iPSC derived cartilage microtissues (iCMTs) and day $21 \mathrm{hPDC}$ derived “callus organoids" (COs) (Figure 5a), COs were seeded into a macro-well (2mm $\varnothing)$ and incubated for 24 hours where after COs were added and fused for additional 24 hours before adding CM1 in ThinCert ${ }^{\mathrm{TM}}$ and CM2 in the well plate (Figure S2a-b, Supporting Information).

\section{Gene expression analysis}

Total RNA from hAC was extracted using the Nucleospin RNA II kit (Macherey-Nagel). Microspheroids from one well were pooled together and total RNA was isolated using QIAshredder (Qiagen) followed by RNeasy Mini Kit (Qiagen). RNA concentration and quality were assessed with NanoDrop 2000 (Thermo Scientific) and PrimeScript ${ }^{\mathrm{TM}}$ RT reagent kit (Takara) was used for cDNA synthesis. Quantitative real-time polymerase chain reaction (qRTPCR) was performed using SYBR ${ }^{\circledR}$ Green (Life Technologies) on Rotor Gene ${ }^{\circledR} 6000$ (Qiagen) and relative differences in expression were calculated using the $2^{-\Delta \Delta \mathrm{Ct}}$ method normalized to the housekeeping gene Glyceraldehyde-3-Phosphate Dehydrogenase (GAPDH) [44]. All protocols were performed according to the manufacturer's protocol.

\section{Subcutaneous implantation}

The zonal constructs were implanted subcutaneously in 8 weeks old female, immune compromised mice (Rj:NMRInu/nu; Jackson laboratory); two samples from different conditions were implanted per mouse. After 4 weeks implantation, explants were taken out and fixed in 4\% PFA for subsequent nanoCT scan and histological analysis. All procedures on animal experiments were approved by the local ethical committee for Animal Research, KU Leuven (P36/2016 ECD) and the animals were housed according to the regulations of the Animalium Leuven (KU Leuven). 


\section{NanoCT imaging and quantification}

After PFA fixation, the explants were washed in PBS and immersed in $20 \%$ Hexabrix $^{\circledR} 320$ (Guerbet Nederland BV) overnight. Hexabrix ${ }^{\circledR} 320$ contains negatively charged ioxaglate molecules which are locally repelled by the negatively charged sulfated glycosaminoglycans (sGAG) containing cartilage enabling visualization of the cartilage tissues [45]. Samples were scanned with nanoCT (Pheonix Nanotom M®, GE Measurement and Control Solutions) at a voltage of $60 \mathrm{kV}$ and a current of $140 \mu \mathrm{A}$ resulting in an isotropic voxel size of $2 \mu \mathrm{m}$ using diamond target, mode 0,500ms exposure time, 1 frame average, 0 image skip, 2400 images and a $0.2 \mathrm{~mm}$ aluminum filter. Region of interest (ROI) was drawn to define total explant volume followed by mineralization quantification using CTAn (Bruker micro-CT, BE) based on automatic Otsu segmentation, 3D space closing and despeckle algorithm. CTvox (Bruker micro-CT, BE) was used to create 3D visualizations.

\section{Histological staining}

After nanoCT imaging, explants were decalcified in ethylenediaminetetraacetic acid (EDTA)/PBS (pH 7.5) for 10 days at $4^{\circ} \mathrm{C}$ followed by paraffin embedding overnight. Explants were sectioned into $5 \mu \mathrm{m}$ slices and de-paraffinized in HistoclearTM (Laborimpex, Belgium) followed by histological staining Hematoxylin-eosin (H\&E), Alcian blue (Sigma; $0.5 \%$ in 1M $\mathrm{HCl}, \mathrm{pH} 1$; counterstain with nuclear fast red (NFR)) and Safranin O (Saf O; Klinipath; 0.25\%; counterstain with fast green and hematoxylin) as previously described [46]. Immunostaining was performed by antigen retrieval $(1 \mathrm{mg} / \mathrm{mL}$ pepsin in $0.02 \mathrm{M} \mathrm{HCl})$, washes, quenching in $3 \%$ $\mathrm{H} 2 \mathrm{O} 2$ and blocking followed by primary antibody incubation overnight at $4{ }^{\circ} \mathrm{C}$ for collagen type II (Col2: dilution 1:50, AB761, Merck Millipore), collagen type I (Col1: dilution 1/200, PA136057, Thermo Fisher), Indian hedgehog (IHH: dilution 1/50, ab80191, Abcam) or human specific osteocalcin (hOCN: dilution 1/5000, gift from E. Van Herck, Legendo, KU Leuven, BE). Next, slides were blocked and incubated with secondary anti-rabbit antibody (dilution 
1:500, 111-035-003, Jackson ImmunoResearch, UK) or anti-Guinea pig antibody (dilution 1:500) followed by visualization with DAB (K3468, Dako, US) and counterstained with haematoxylin. Stained sections were imaged with Leica M165 FC microscope (Microsystems, $\mathrm{BE})$.

\section{Histomorphometry}

The number of cells $/ \mathrm{mm}^{2}$ was quantified on Alcian Blue stained histological sections of the in vitro hierarchical constructs using ImageJ [47,48]. Briefly, images were deconvoluted using "Alcian blue \& H" and the color_2 representing the nuclei was chosen. This image was made binary, followed by watershed and particle analysis $\left(5-500 \mu \mathrm{m}^{2}\right)$ to quantify the number of particles (cells) in the chosen region (3 sections per condition; top and bottom were quantified). The Saf O stained area of in vivo samples was quantified using Ilastik version 1.3.3post3 pixel classification [49] and ImageJ [48].The Ilastik software was trained to recognize Saf O stained regions based on color/intensity on three different samples. Subsequently, three distributed sections per sample were analyzed and HDF5 files were opened in ImageJ to define \% of Saf O stained area ("number Saf O stained pixels" / "number sample pixels" defined by histogram).

\section{Scanning electron microscopy and Raman spectrometry}

Samples were sectioned into $5 \mu \mathrm{m}$ slices and de-paraffinized in HistoclearTM (Laborimpex, Belgium) followed by de-hydration. Samples to be imaged with scanning electron microscopy (SEM) were sputter coated with gold particles (EDWARDS S150) and subsequently imaged with Nova NanoSEM ${ }^{\mathrm{TM}} 450$ (FEI). Non-polarized Raman spectra were collected by a T64000 micro-Raman system (Horiba). The laser wavelength excitation selected was the $514.5 \mathrm{~nm}$ emitted from an SSD laser and the excitation beam was focused on the deparaffinated $5 \mu \mathrm{m}$ thin cross-sections by a 100x microscope objective. Confocal configuration was used in order to minimize the spectral contribution of the microscope glass slide. The scattered beam passed through an appropriate edge filter and was then focused on the entrance slit of a single 
spectrograph $(600 \mathrm{~g} / \mathrm{mm})$. The signal was detected by a 2D CCD detector with a resolution of $\sim 7 \mathrm{~cm}^{-1}$. For statistical purposes, a series of more than 6 spectra was collected from each sample.

\section{Statistical analysis}

All experiments were performed with at least three samples per condition. Statistical analysis was performed using GraphPad Prism 8 software (GraphPad Software, Inc., La Jolla, CA) and represented as mean \pm SD if nothing else was stated. One-way or two-way Analysis of Variance (ANOVA) followed by Student's t test or Tukey's post-hoc test respectively, was used to find the significant differences between the means of the different groups with $\mathrm{p}<0.05$ : ${ }^{*}, \mathrm{p}<0.01$ : $* *$ and $\mathrm{p}<0.001: * * *$.

\section{Results}

\subsection{Human periosteum derived cells form microtissues with cartilaginous and} hypertrophic phenotype

hPDCs were seeded into non-adherent microwells to form microspheroids of homogenous size $(107 \pm 12 \mu \mathrm{m}$ dimeter, 2700 microspheroids quantified day 1). The microspheroids were differentiated in chemically defined chondrogenic media for 21 days leading to accumulation of extracellular matrix (Figure 1a and Figure S1a-c, Supporting Information). Proliferation assay demonstrated high presence of proliferating cells at day $7(53 \pm 21 \%)$ followed by a significant decrease on day 14 and 21 (Figure 1b-c) and both viable and dead cells were present throughout the differentiation process (Figure S1d, Supporting Information). Gene expression analysis was performed on the different building block populations before assembly to define the maturity of microspheroids over time. mRNA expression of the early chondrogenic marker SOX9 was up-regulated 7-folds after 7 days in culture without significant change on day 14 followed by a 3-fold down-regulation at day 21 (Figure 1d). The mRNA of the gene COL2A1, encoding the alpha-1 chain of type II collagen present in cartilage, was up-regulated 1600-fold 
by day 14 followed by a significant up-regulation of 5200-fold after 21 days in culture. Upregulation of the (pre)hypertrophic gene markers $I H H$ and $C O L X$ was detected after 21 days in culture (Figure 1e). These results indicate the formation of chondrogenic microtissues after 7 to 14 days, which attain a (pre)hypertrophic phenotype after 21 days in culture corroborating our previously attained data within the same culture platform, where the day 21 microtissues were defined as "callus organoids" (COs) [19].

\subsection{Building blocks from adult progenitor cells form hierarchical structures for gradient cartilaginous tissue}

The osteochondral tissue is defined by layers of tissues with different properties going from avascular cartilage, mineralized cartilage to subchondral bone. To create a hierarchical intermediate tissue that upon implantation would create these layers, building blocks of different maturity were sequentially assembled in a non-adherent macro-well of $2 \mathrm{~mm}$ diameter. Day 7 and day 14 microtissues were added to represent the chondrogenic part, followed by the prehypertrophic day 21 microtissues expected to continuously mature and mineralize in vivo to represent the bone part. The macro-well was placed in a Thincert ${ }^{\mathrm{TM}}$ to allow media diffusion from both the top and bottom of the construct during the 24 hours fusion period. Histological characterization of the in vitro construct showed a gradient increase in Alcian blue staining, Saf $\mathrm{O}$ and collagen type II (Figure 1f-h). Day 7 microtissues in the bottom layer contained a higher number of cells per area $\left(4062 \pm 463\right.$ cells $\left./ \mathrm{mm}^{2}\right)$ compared to the top layer with day 21 microtissues $\left(2538 \pm 521\right.$ cells $\left./ \mathrm{mm}^{2}\right)$ while the top layer contained increased sulfated glycosaminoglycans (sGAG) and collagen type II positive areas confirming the formation of a hierarchical construct.

Next, the constructs were implanted subcutaneously in immunocompromised mice for 4 weeks to evaluate their in vivo tissue formation/maturation potency. Alcian blue staining indicated the formation of two distinct layers, a fibrocartilage-like layer which was non- 
mineralized and a second layer containing mature hypertrophic chondrocyte-like cells (Figure S1e, Supporting Information). Collagen type I immunostaining (COL1) demonstrated positive areas in the mineralized part but also in the fibrocartilage-like layer, further indicating a fibrous nature of the non-mineralized region (Figure S1f, Supporting Information). NanoCT demonstrated that the top layer was mineralized (Figure 1k) and certain areas were positive for fast green (Figure 1i), hOCN (Figure 1j) and fluorescent H\&E staining (Figure S1g, Supporting Information) indicating occurrence of bone formation. Although a layered structure was created after subcutaneous in vivo implantation, the cartilaginous part showed a fibrous phenotype with elongated cells, indicating that the microtissues were insufficient for formation of Saf O positive cartilage at a subcutaneous implantation site. However, as previously described, the (pre)hypertrophic day 21 microtissues, "callus organoids" (COs), showed promise for bone formation.

\subsection{Chondroprogenitors from human induced pluripotent stem cells form cartilage microtissues in vitro}

Since the day 7 and 14 microtissues formed fibrocartilage in vivo, an alternative cell source for creation of the cartilaginous part was introduced. iPSCs have emerged as a promising cell source for cartilage TE and Saf $\mathrm{O}$ positive cartilage tissue in vivo has successfully been produced from human iPSCs [36-38]. Here, human iPSCs were differentiated into highly Saf O positive chondrogenic nodules (approximately $2 \mathrm{~mm}$ in diameter) (Figure 2a) [37]. Cells within the nodules were subsequently isolated by enzymatic digestion resulting in a yield of $256500 \pm 37590$ iPSC-derived chondrocytes (iChon) per nodule (140 nodules from 4 independent digestions). Gene expression of three independent iChon batches were compared to hACs from three donors to assess their chondrogenic phenotype. iChon had increased mRNA expression of the early chondrogenic marker $S O X 9$ and $C O L 2$ but no significant difference was seen for ACAN and COLl (Figure 2b). These data demonstrate that iPSCs were successfully 
differentiated into chondrocytes which secreted sGAG-rich cartilaginous matrix and isolated iChon.

Next, the isolated chondroprogenitors (iChon) were seeded into non-adherent microwells. The entrapped chondroprogenitors underwent self-aggregation and subsequent condensation into microspheroids at day 7 (Figure 2c) followed by an increase in size over time (Figure 2d). Alcian blue and Saf $\mathrm{O}$ staining demonstrated presence of extracellular matrix already at day 7 (Figure 2e-f). A down-regulation of the early chondrogenic marker SOX9 (Figure 2g) was detected in the building blocks as compared to the isolated iPSC derived chondrocytes (iChon; Day 0), indicating an effect of the aggregation and microspheroid culture. However, the chondrogenic marker COL2 remained stable over time (Figure 2g) suggesting that collagen type II secretion remained stable. In addition, the genes GREMI and FRZB related to articular cartilage maintenance were 3- and 4.5-fold up-regulated, respectively, after 21 days (Figure 2g) while COL1 gene expression associated with fibrocartilage also was up-regulated (Figure 2h) [50]. Furthermore, no significant difference in gene expression of the hypertrophic markers $I H H$ or $P T H 1 R$ was detected between day 0 and 21, although significant up-regulation of the hypertrophic genes $C O L X$ (3-fold), ALP (2-fold) and LEF1 (3.5-fold) was detected on day 21 (Figure 2i). In conclusion, isolated iChon were able to form cartilage-like microtissues with increasing size over time and up-regulation of articular cartilage gene markers, although certain hypertrophic gene markers also were up-regulated on day 21.

\subsection{Chondrogenic and prehypertrophic building blocks assemble into integrated constructs in vitro}

The presented results indicate that the day 21 COs (Figure 1d-e) and day 21 iCMTs (Figure 2) have characteristics suitable to create a dual construct from the bottom up with one part (iCMTs) representing cartilage and the second part (COs) more hypertrophic cartilage potentially serving as a template for the subchondral bone. Indeed, gene expression was analyzed to further assess 
the cellular phenotype of the distinct building blocks. No significant difference of the chondrogenic gene markers SOX9 and COL2 was detected between day 21 hPDC- and iChonderived building blocks while mRNA levels of the genes GREMI and FRZB associated with articular cartilage maintenance were significantly higher in iChon compared to hPDC building block on day 21 (Figure 3a). Furthermore, the mRNA levels of hypertrophic gene markers were significantly higher in the hPDC-derived building blocks compared to iChon-derived building blocks on day 21: IHH (37-fold), COLX (781-fold), ALP (601-fold), PTH1R (14-fold) and LEF1 (2-fold) (Figure $3 \mathrm{~b}$ and Figure S2c, Supporting Information). No significant difference in COL1 mRNA levels between hPDC- and iChon-derived building blocks were observed on day 21 (Figure S2d, Supporting Information).

Since formation of larger constructs requires fusion of building blocks, quantification of fusion capacity was assessed by fusion of two building blocks of each tissue type (Figure 3c). 24 hours fusion was previously shown to be suitable for assembly of COs which was confirmed by the significantly lower aspect ratio (AR) already observed after $10 \mathrm{hrs}$ after which it continued to decrease (Figure 3d). Markedly, iCMTs fused (Supplemental Movie 1) significantly slower and consequently a longer fusion time for iCMTs was used. The iCMTs were assembled in a non-adherent macro-well ( $2 \mathrm{~mm}$ diameter) which resulted in a thin layer (Figure S2a, Supporting Information). After $24 \mathrm{hrs,} \mathrm{COs} \mathrm{were} \mathrm{added} \mathrm{on} \mathrm{top} \mathrm{for} \mathrm{an} \mathrm{additional}$ 24 hrs of fusion into a dual construct (Figure 3f, Figure S2b, Supporting Information). Hence, to correlate with the AR quantification, iCMTs were fused for $48 \mathrm{hrs}$ in total while the COs were fused for $24 \mathrm{hrs}$.

Gene expression analysis demonstrated a distinct difference between hPDC and iChon building blocks on day 21 (Figure 3a-b) and vague differences were further detected with histological staining. Alcian blue staining of fused constructs demonstrated presence of ECM although no or small differences were detected between COs and iCMTs (Figure 4a), while Saf O staining was more prominent in iCMTs (Figure 4b) and IHH immunostaining hinted towards 
increased staining of cells within COs (Figure 4c, black arrows). Next, SEM was performed on the different building blocks (CO and iCMT) to visualize the ECM structure with high magnification (Figure 4d). As comparison, the human articular cartilage on an osteochondral section (Figure S2e, Supporting Information) was imaged. CO exhibited a dense fibrous ECM structure (Figure 4d, CO 50 000x) while the ECM structure of iCMT was more porous, similar to the structures detected in articular cartilage.

To further elucidate the chemical composition of ECM, Raman spectroscopy was applied on fused in vitro constructs (COs and iCMTs). Raman spectroscopy has previously been used to assess the quality of tissue engineered cartilage as well as native articular cartilage [5153]. Typical Raman spectra from native cartilage tissue as well as engineered cartilaginous tissues (hPDC derived COs and iChon derived iCMTs) are depicted in Figure 4e. Several bands may be resolved via assignment found in the literature [52,53]. The most prominent bands are the ones characteristically described amide I and III bands at $\sim 1650$ and $1250 \mathrm{~cm}^{-1}$ respectively and the $\mathrm{CH}_{2}$ and $\mathrm{CH}_{3}$ bending bands at $\sim 1450 \mathrm{~cm}^{-1}$. The latter are ascribed primarily to collagen chemical species, however respective bands of lipids may also contribute in the same spectral range. Two additional weaker bands at $\sim 1060$ and $1130 \mathrm{~cm}^{-1}$ have been used in order to predict the amount of glycosaminoglycans (GAG) and Aggrecan in the samples [54,55]. One of the foremost arguments resulting from Raman spectroscopy is the fact that the intensity of the aliphatic bands is enhanced in both cases of engineered tissues. The intensity enhancement is revealed by comparison with the amide I and amide III bands in the respective spectra. An additional finding is that the Raman prediction of GAG and Aggrecan for the two engineered cartilaginous tissues differs (Figure 4f). 


\subsection{Integrated constructs can maintain maturation properties in vivo and form osteochondral-like tissues}

The fused constructs (only COs or iCMTs and Dual) were implanted subcutaneously for 4 weeks to evaluate their capacity to mature in vivo (Figure 5a). Mineralization was detected in both dual and osteogenic (COs) constructs but not in chondrogenic (iCMTs) constructs (Figure 5b and Figure S3a, Supporting Information). As expected, the dual constructs contained significantly less mineralization $(18 \pm 6 \%)$ as compared to the hPDC constructs $(29 \pm 3 \%)$ (Figure 5g and Figure S3b, Supporting Information). To enable visualization of cartilaginous tissue with nanoCT, the contrast agent Hexabrix ${ }^{\circledR}$ (ioxaglate meglumine $\&$ ioxaglate sodium) was used. Ionic Hexabrix ${ }^{\circledR}$ is detected by nanoCT and repelled by negatively charged tissue, such as sGAG-rich cartilage, resulting in decreased gray values for cartilaginous tissues [45]. Cartilaginous tissue was detected in both the iCMTs (3/3 samples) and dual constructs (3/6 samples). 3D rendering of mineralized and cartilaginous tissue demonstrated that the two parts formed a dual tissue (Figure 5b).

$H \& E$ staining of the dual constructs indicated areas of integration between the distinct building blocks (Figure 5c, black arrow) although non-mineralized fibrous regions were also discerned between the two parts (Figure 5c, white arrow and Figure S3d, Supporting Information). The presence of two distinct zones was further demonstrated with positive Saf O staining in three of six dual samples (Figure 5d and Figure S3d, Supporting Information) as well as collagen type II positive areas in the iCMT construct/part (Figure 5e). However, COs in dual samples with advanced maturation into bone ossicles, containing cortical bone and bone marrow (Figure S3e, Supporting Information), appeared to subdue the iCMTs. Quantification of the Saf O positive area showed that $3 / 6$ dual implants contained $41 \pm 5 \%(n=3)$ while the other 3/6 dual implants did not contain Saf O positive areas (Figure 5h). Interestingly, 2/3 iCMT implants generated homogeneous Saf O positive tissues, demonstrating that the assembled building blocks were able to fuse into one tissue without evident microtissue structures after 
implantation (Figure 5d: iCMTs). Furthermore, 2/5 CO implants generated tissues with Saf O positive areas indicating the presence of hypertrophic cartilage that was not yet fully remodeled (Figure 5d and h, COs).

Human osteocalcin (hOCN) positive areas in the $\mathrm{CO}$ construct/part provided evidence that at least some of the implanted human cells were contributing to bone formation (Figure 5f) and COL1 immunostaining demonstrated higher positivity in $\mathrm{CO}$ constructs/parts, while presence of COL1 in the iCMT explants was mainly present in the fibrous periphery (Figure S3f, Supporting Information). Structure thickness (St. Th.) and structure linear density (St.Li.Dn) within the mineralized tissue structures (Figure S3c, Supporting Information) of COs and Dual constructs did not demonstrate any significant differences (Figure 5i-j). Variations in mineral structure thickness and structure linear density may instead be attributed to the bone maturation (Figure 5i, empty data points represent constructs without Saf O positive regions). These data demonstrated that the different building blocks could integrate into dual constructs with preserved maturation properties to form osteochondral-like tissues upon subcutaneous implantation. However, the variable in vivo outcome demonstrated that the subtle balance required between the two tissues was not fully achieved.

\section{Discussion}

In this study, we aimed to engineer a patterned osteochondral tissue using distinct cartilaginous building blocks, which allowed the progressive formation of an osteochondral unit upon subcutaneous in vivo implantation. The native osteochondral tissue consists of a gradient with multiple cell types, which maintain a stability of the full depth joint surface including nonmineralized hyaline cartilage, mineralized cartilage and subchondral bone. The one-module culture set-up currently used in TE is limiting the complexity of TE constructs that can be produced [56]. A multimodular approach using microtissues and organoids [30,57] was therefore proposed here, to allow the assembly of multiple building blocks into designed 
gradients corresponding to the native structure. By generating a hierarchical tissue assembly from building blocks of defined developmental stages (proliferation to hypertrophy) we aimed to create an engineered implant able to form an osteochondral unit in vivo. However, although mineralized tissue was detected, the hPDC derived microtissues with chondrogenic gene expression phenotype (SOX9, COL2A1 up-regulated mRNA expression) resulted in fibrocartilage upon subcutaneous implantation (Figure 1i-j and S1e-f, Supporting Information). The use of multipotent MSCs has been shown to result in fibrocartilage or onset of hypertrophy leading to mineralization after long term implantation, even at orthotopic sites [25,34], indicating that adult MSCs either do not have the intrinsic capacity to form stable cartilage or that current chondrogenic differentiation protocols are not appropriate. For further improvement, developmentally-inspired expansion and differentiation protocols have been presented to obtain more stable cartilage from adult progenitor cells $[58,59]$.

In this study, we aimed at using human iPSCs as cell source for the generation of cartilage microtissues which could result in Saf $\mathrm{O}$ positive cartilage after subcutaneous implantation. In contrast to hACs, chondrocytes derived from iPSCs can be produced in large amounts without the need of making additional cartilage defects for obtaining biopsies, making iPSCs potentially attractive for clinical translation [60]. Additionally, iPSC-derived microtissues can be produced through protocols ensuring homogeneity and purity $[36,37,61]$ and their production has scale-up potential by using bioreactors [62]. We demonstrated that cells isolated from chondrogenic nodules derived from iPSCs (iChon) attained comparable gene expression levels of $A C A N$ and COL1A1 as hACs while SOX9 and COL2 mRNA transcript levels were higher in iChon (Figure 2b). The relatively high SOX9 and COL2 mRNA expression in iChon compared to hACs may be linked to a younger cell phenotype of iChon or be an effect of the well-known event of dedifferentiation during monolayer culture [63,64]. Subsequent aggregation of iChon into microspheroids allowed the formation of cartilage microtissues (iCMTs) permitting bottom-up formation of larger constructs. Upon implantation, the iCMT 
constructs formed uniform cartilaginous tissues, demonstrating building block integration (Figure 5). This is in contrast to previously published data of assembled iPSC cartilage nodules [37] and the improved building block integration could be related to the relatively short culture time for iChon as microtissues (3 weeks) compared to nodules (6-8 weeks). This timedependent fusion capacity is a phenomenon previously described for sheep articular chondrocytes [65]. This is an interesting finding as compared to prior art [66] and the reconstitution of iPSC pellets into iChon microtissues could be a methodology enabling module fusion for modular tissue engineering of cartilage.

Although iPSCs demonstrate promise for cartilage regeneration, few studies have applied iPSC-derived chondrocytes to tissue engineer zonal osteochondral grafts, to our knowledge. Lin et.al. demonstrated the formation of an osteochondral unit in vitro using iPSCs for the purpose of drug screening but no in vivo functionality was reported [67]. Our approach involved the combination of iChon derived iCMTs for the cartilage part to overcome limitations using hACs and adult progenitor cell (hPDC) derived COs for the bone part to take advantage of their robust endochondral bone forming potency [19]. Gene expression demonstrated a distinct cellular difference between COs and iCMTs (Figure 3a-b) and Raman spectroscopy gave information regarding ECM composition (Figure 4e-f). Engineered iCMTs showed higher resemblance to native cartilage in terms of the proportional GAG and aggrecan presence to overall collagen (as quantified through the main Amide I peak) as indicated in the Raman spectra analysis. The presence of lower aggrecan in the case of hPDC engineered COs could also be linked to a more hypertrophic phenotype as seen also in the context of osteoarthritis [68]. These findings provide additional information on the properties of the extracellular matrix that are in accordance with the gene expression analysis and immunohistochemistry carried out between the different conditions, further distinguishing the properties of the extracellular matrix of the two populations of cartilaginous microtissues. With further validation, Raman spectra could be used to quantitatively identify engineered tissue quality properties (tissue identity) 
with high precision and hopefully act as predictive tools for implant potency and novel biomarker identification for organoid-based implants $[52,53]$. However, it should be noted that the measurements in our work were carried out in fixated histological sections hence further validation in "living organoids" should be carried out in future experiments.

Subcutaneous implantation of the assembled constructs demonstrated tissue maturation and the two different zones (COs and iCMTs) showed the development of a tissue interface that appeared to have areas of integration (Figure 5c, black arrow), quite critical for its potential biomechanical and biological behavior in vivo when implanted in a deep osteochondral defect. Nevertheless, half of the dual implants in our study generated both Saf O positive and mineralized regions while the other half lacked Saf O positive cartilaginous regions. These results highlight the importance of a balanced cellular phenotype between the cartilaginous and bony part. The absence of a cartilaginous region could be attributed to the up-regulation of certain hypertrophic gene markers in the iCMTs at day 21 (Figure 2i: COLX, ALP, LEF1). In addition, the mineralized part in the dual constructs containing Saf O positive regions was less mature compared to samples without Saf O positive regions, indicating that the maturation of the COs bone part overpowered the maintenance of the cartilaginous iCMT phenotype.

The native cartilage-bone interface of the joint surface is defined by the tidemark, a tissue junction between mineralized and non-mineralized cartilage, which plays an important role in the maintenance of a balanced osteochondral unit [69,70]. Attempts to recreate an in vitro cartilage-bone interface using combinations of biomaterials and cells have been presented in the literature, as well as scaffold-free approaches to recreate the crucial mineralized cartilage zone between the non-mineralized cartilage and bone substitute $[9,71,72]$. Introduction of a biomaterial- or protein-based barrier, i.e. electrospun membrane [73] or noggin [24] respectively, between iCMTs and COs may be a solution to further improve the in vivo outcome. However, the formation of the tidemark is hypothesized to be linked to mechanical stimulation $[74,75]$ and orthotopic implantation is therefore probably necessary to evaluate this point. 
Hence, orthotopic implantation in an osteochondral defect model is also required to assess whether the interface between iCMTs and OCs could possess a functional role corresponding to that of the tidemark. Moreover, the building block approach applied in our work provides the possibility to model cartilage-bone integration by detailed analysis of building block fusion as previously described for hepato-biliary-pancreatic organoids [76]. The in vitro building block fusion could further be combined with novel microwell platform designs, which might elicit improvements in micro-engineering of osteochondral niches in vitro [77]. Additionally, the development of dual constructs with an iPSC-derived bone part $[36,78,79]$ is envisaged to create an osteochondral graft entirely derived from iPSCs.

The modular approach suggested in this work is amenable to the use of recently developed biofabrication technologies for a more automated and guided bioassembly process [9,57,80-82]. The use of large spheroids for bioprinting has been exhibited [82] for the formation of spatially organized tissues [81], in the presence of scaffolds. The intrinsic design of scaffolds could also be designed to further promote a gradient tissue formation [83] in addition to deposition of spheroids. Recently, aspiration assisted bioprinting showed that single microspheroids, of equivalent size to our study, could be picked-up and transferred at desired locations [80]. Currently, the throughput of these strategies is low and considerable time would be needed for the biomanufacturing of clinically relevant tissue volumes. However, it is plausible that through parallelization and further intensification of these processes it could be possible to automate the production of complex patterned osteochondral tissues as developed in the present study.

\section{Conclusion}

Organoids engineered in vitro possess the capacity to semi-autonomously undergo maturation and developmental-like processes in vivo. This provides the unique opportunity to build complex living implants within which a quasi-deterministic behavior can be engineered. In this 
study, we embedded genetically distinct populations of cartilaginous tissue intermediates within a single implant in order to create an osteochondral tissue unit. iCMTs, derived from human iPSCs, were assembled with COs, derived from human PDCs, to engineer a zonal structure. Upon implantation, the different zones (iCMTs and COs) of the implant, could result in the formation of a dual structure of cartilage and bone corresponding to the preimplantation design. Additional studies to further stabilize dual maturation as well as implantation at orthotopic sites will be needed to explore the capacity of these implants to gradually integrate at the defect site and result in an osteochondral tissue.

\section{Conflicts of interest}

The authors declare that they have no competing interests.

\section{Author contributions}

Gabriella Nilsson Hall: Conceptualization, Methodology, Investigation, Project administration, Writing - Original Draft. Wai Long Tam: Conceptualization, Methodology, Investigation, Writing - Review \& Editing. George A. Voyiatzis and Konstantinos S. Andrikopoulos: Methodology, Investigation, Writing - Review \& Editing. Leire CasasFraile: Methodology, Investigation, Writing - Review \& Editing. Liesbet Geris: Conceptualization, Writing - Review \& Editing. Frank P. Luyten: Conceptualization, Supervision, Writing - Review \& Editing. Ioannis Papantoniou: Conceptualization, Supervision, Writing - Original Draft.

\section{Acknowledgements}

F.P.L. and I.P. contributed equally to this work. Kathleen Bosmans is thanked for performing in vivo experiments; Inge Van Hoven, Samuel Ribeiro Viseu and Tim Reekmans for their experimental assistance. Research was funded by the Research Foundation Flanders (FWO) G.N.H: 1S05116N, I.P.: 12O7916N, and CARTiPLEX: G0A4718N, the European Research 
Council under the European Union's Seventh Framework Program (FP/2007-2013)/ERC (249191) and Horizon 2020 Framework Program (H2020/2014-2021)/ERC (772418), the special research fund of the KU Leuven (GOA/13/016 and C24/17/077) and BONE Interreg North West Europe (NWE 497), European Regional Development Fund and JointPromise (H2020-EU.3.1.3; 874837). This work was supported by the partners of Regenerative Medicine Crossing Borders (http://www.regmedxb.com). Powered by EWI Flanders. The SEM images were generated on Nova NanoSEMTM 450 by Tom Van der Donck at the "Electron Microscopy" facility, Department of Materials Engineering (MTM), KU Leuven. The micro (or nano)-CT images were generated on the X-ray computed tomography facility of the Department of Development and Regeneration of the KU Leuven, financed by the Hercules Foundation (project AKUL/13/47). The funders had no role in study design, data collection and analysis, decision to publish, or preparation of the manuscript. This work is part of Prometheus, the KU Leuven R\&D division for skeletal tissue engineering (http://www.kuleuven.be/prometheus).

\section{Data Availability}

Data will be made available on request.

\section{References}

[1] T. Gorbachova, Y. Melenevsky, M. Cohen, B.W. Cerniglia, Osteochondral lesions of the knee: Differentiating the most common entities at MRI, Radiographics. 38 (2018) 14781495. doi:10.1148/rg.2018180044.

[2] T.D. Brown, R.C. Johnston, C.L. Saltzman, J.L. Marsh, J.A. Buckwalter, Posttraumatic osteoarthritis: A first estimate of incidence, prevalence, and burden of disease, in: J. Orthop. Trauma, 2006. doi:10.1097/01.bot.0000246468.80635.ef.

[3] G. Filardo, L. Andriolo, F. Soler, M. Berruto, P. Ferrua, P. Verdonk, F. Rongieras, D.C. Crawford, Treatment of unstable knee osteochondritis dissecans in the young adult: results and limitations of surgical strategies - The advantages of allografts to address an 
osteochondral challenge, Knee Surgery, Sport. Traumatol. Arthrosc. 27 (2019) 17261738. doi:10.1007/s00167-018-5316-5.

[4] A. Pareek, T.L. Sanders, I.T. Wu, D.R. Larson, D.B.F. Saris, A.J. Krych, Incidence of symptomatic osteochondritis dissecans lesions of the knee: a population-based study in Olmsted County, Osteoarthr. Cartil. $25 \quad$ (2017) 1663-1671. doi:10.1016/j.joca.2017.07.005.

[5] R.J. Lories, F.P. Luyten, The bone-cartilage unit in osteoarthritis, Nat. Rev. Rheumatol. 7 (2011) 43-49. doi:10.1038/nrrheum.2010.197.

[6] T. Ogura, G. Merkely, T. Bryant, C.S. Winalski, T. Minas, Autologous Chondrocyte Implantation "Segmental-Sandwich" Technique for Deep Osteochondral Defects in the Knee: Clinical Outcomes and Correlation With Magnetic Resonance Imaging Findings, Orthop. J. Sport. Med. 7 (2019) 232596711984717. doi:10.1177/2325967119847173.

[7] K.T.A. Lambers, J. Dahmen, M.L. Reilingh, C.J.A. van Bergen, S.A.S. Stufkens, G.M.M.J. Kerkhoffs, No superior surgical treatment for secondary osteochondral defects of the talus, Knee Surgery, Sport. Traumatol. Arthrosc. 26 (2018) 2158-2170. doi:10.1007/s00167-017-4629-0.

[8] J. Visser, D. Gawlitta, K.E.M. Benders, S.M.H. Toma, B. Pouran, P.R. van Weeren, W.J.A. Dhert, J. Malda, Endochondral bone formation in gelatin methacrylamide hydrogel with embedded cartilage-derived matrix particles, Biomaterials. 37 (2015) 174-182. doi:10.1016/j.biomaterials.2014.10.020.

[9] P. Diloksumpan, M. de Ruijter, M. Castilho, U. Gbureck, T. Vermonden, P.R. van Weeren, J. Malda, R. Levato, Combining multi-scale 3D printing technologies to engineer reinforced hydrogel-ceramic interfaces, Biofabrication. 12 (2020) 025014. doi:10.1088/1758-5090/ab69d9.

[10] R.A. Vindas Bolaños, S.M. Cokelaere, J.M. Estrada McDermott, K.E.M. Benders, U. Gbureck, S.G.M. Plomp, H. Weinans, J. Groll, P.R. van Weeren, J. Malda, The use of a 
cartilage decellularized matrix scaffold for the repair of osteochondral defects: the importance of long-term studies in a large animal model, Osteoarthr. Cartil. 25 (2017) 413-420. doi:10.1016/j.joca.2016.08.005.

[11] E.J. Sheehy, T. Vinardell, C.T. Buckley, D.J. Kelly, Engineering osteochondral constructs through spatial regulation of endochondral ossification, Acta Biomater. 9 (2013) 5484-5492. doi:10.1016/j.actbio.2012.11.008.

[12] C. Stüdle, Q. Vallmajó-Martín, A. Haumer, J. Guerrero, M. Centola, A. Mehrkens, D.J. Schaefer, M. Ehrbar, A. Barbero, I. Martin, Spatially confined induction of endochondral ossification by functionalized hydrogels for ectopic engineering of osteochondral tissues, Biomaterials. 171 (2018) 219-229. doi:10.1016/j.biomaterials.2018.04.025.

[13] P.J. Stiers, S. Stegen, N. van Gastel, R. Van Looveren, S. Torrekens, G. Carmeliet, Inhibition of the Oxygen Sensor PHD2 Enhances Tissue-Engineered Endochondral Bone Formation, J. Bone Miner. Res. 34 (2019) 333-348. doi:10.1002/jbmr.3599.

[14] A. Lang, M. Kirchner, J. Stefanowski, M. Durst, M.C. Weber, M. Pfeiffenberger, A. Damerau, A.E. Hauser, P. Hoff, G.N. Duda, F. Buttgereit, K. Schmidt-Bleek, T. Gaber, Collagen I-based scaffolds negatively impact fracture healing in a mouse-osteotomymodel although used routinely in research and clinical application, Acta Biomater. 86 (2019) 171-184. doi:10.1016/j.actbio.2018.12.043.

[15] H. Katagiri, L.F. Mendes, F.P. Luyten, Reduction of BMP6-induced bone formation by calcium phosphate in wild-type compared with nude mice, J. Tissue Eng. Regen. Med. 13 (2019) 846-856. doi:10.1002/term.2837.

[16] P. Lenas, M. Moos, F.P. Luyten, Developmental engineering: a new paradigm for the design and manufacturing of cell-based products. Part I: from three-dimensional cell growth to biomimetics of in vivo development., Tissue Eng. Part B. Rev. 15 (2009) 38194. doi:10.1089/ten.TEB.2008.0575.

[17] P. Lenas, F.P. Luyten, An Emerging Paradigm in Tissue Engineering: From Chemical 
Engineering to Developmental Engineering for Bioartificial Tissue Formation through a Series of Unit Operations that Simulate the In Vivo Successive Developmental Stages $\uparrow$, Ind. Eng. Chem. Res. 50 (2011) 482-522. doi:10.1021/ie100314b.

[18] C. Scotti, E. Piccinini, H. Takizawa, A. Todorov, P. Bourgine, A. Papadimitropoulos, A. Barbero, M.G. Manz, I. Martin, Engineering of a functional bone organ through endochondral ossification., Proc. Natl. Acad. Sci. U. S. A. 110 (2013) 3997-4002. doi:10.1073/pnas.1220108110.

[19] G. Nilsson Hall, L.F. Mendes, C. Gklava, L. Geris, F.P. Luyten, I. Papantoniou, Developmentally Engineered Callus Organoid Bioassemblies Exhibit Predictive In Vivo Long Bone Healing, Adv. Sci. 7 (2020) 1-16. doi:10.1002/advs.201902295.

[20] E. Farrell, S.K. Both, K.I. Odörfer, W. Koevoet, N. Kops, F.J. O’Brien, R.J.B. De Jong, J.A. Verhaar, V. Cuijpers, J. Jansen, R.G. Erben, G.J.V.M. Van Osch, In-vivo generation of bone via endochondral ossification by in-vitro chondrogenic priming of adult human and rat mesenchymal stem cells, BMC Musculoskelet. Disord. 12 (2011) 31. doi:10.1186/1471-2474-12-31.

[21] R.S. Decker, Articular cartilage and joint development from embryogenesis to adulthood, Semin. Cell Dev. Biol. 62 (2017) 50-56. doi:10.1016/j.semcdb.2016.10.005.

[22] E.J. Mackie, L. Tatarczuch, M. Mirams, The skeleton: a multi-functional complex organ: the growth plate chondrocyte and endochondral ossification., J. Endocrinol. 211 (2011) 109-121. doi:10.1530/JOE-11-0048.

[23] J. Li, H. Luo, R. Wang, J. Lang, S. Zhu, Z. Zhang, J. Fang, K. Qu, Y. Lin, H. Long, Y. Yao, G. Tian, Q. Wu, Systematic Reconstruction of Molecular Cascades Regulating GP Development Using Single-Cell RNA-Seq, Cell Rep. 15 (2016) 1467-1480. doi:10.1016/j.celrep.2016.04.043.

[24] A. Ray, P.N.P. Singh, M.L. Sohaskey, R.M. Harland, A. Bandyopadhyay, Precise spatial restriction of BMP signaling is essential for articular cartilage differentiation, Dev. 142 
(2015) 1169-1179. doi:10.1242/dev.110940.

[25] L.F. Mendes, H. Katagiri, W.L. Tam, Y.C. Chai, L. Geris, S.J. Roberts, F.P. Luyten, Advancing osteochondral tissue engineering: Bone morphogenetic protein, transforming growth factor, and fibroblast growth factor signaling drive ordered differentiation of periosteal cells resulting in stable cartilage and bone formation in vivo, Stem Cell Res. Ther. 9 (2018) 1-13. doi:10.1186/s13287-018-0787-3.

[26] S. Critchley, G. Cunniffe, A. O’Reilly, P. Diaz-Payno, R. Schipani, A. McAlinden, D. Withers, J. Shin, E. Alsberg, D.J. Kelly, Regeneration of Osteochondral Defects Using Developmentally Inspired Cartilaginous Templates, Tissue Eng. - Part A. 25 (2019) 159_ 171. doi:10.1089/ten.tea.2018.0046.

[27] I. Gadjanski, G. Vunjak-Novakovic, Challenges in engineering osteochondral tissue grafts with hierarchical structures, Expert Opin. Biol. Ther. 15 (2015) 1583-1599. doi:10.1517/14712598.2015.1070825.

[28] Y. Shwartz, S. Viukov, S. Krief, E. Zelzer, Joint Development Involves a Continuous Influx of Gdf5-Positive Cells, Cell Rep. $15 \quad$ (2016) 2577-2587. doi:10.1016/j.celrep.2016.05.055.

[29] E. Kunisch, A.K. Knauf, E. Hesse, U. Freudenberg, C. Werner, F. Bothe, S. Diederichs, W. Richter, StarPEG/heparin-hydrogel based in vivo engineering of stable bizonal cartilage with a calcified bottom layer, Biofabrication. 11 (2019) 015001. doi:10.1088/1758-5090/aae75a.

[30] B.K. Babur, K. Futrega, W.B. Lott, T.J. Klein, J. Cooper-White, M.R. Doran, Highthroughput bone and cartilage micropellet manufacture, followed by assembly of micropellets into biphasic osteochondral tissue, Cell Tissue Res. 361 (2015) 755-768. doi:10.1007/s00441-015-2159-y.

[31] L. Ouyang, J.P.K. Armstrong, M. Salmeron-Sanchez, M.M. Stevens, Assembling Living Building Blocks to Engineer Complex Tissues, Adv. Funct. Mater. 1909009 (2020) 1- 
22. doi:10.1002/adfm.201909009.

[32] S. Bhumiratana, R.E. Eton, S.R. Oungoulian, L.Q. Wan, G. a Ateshian, G. VunjakNovakovic, Large, stratified, and mechanically functional human cartilage grown in vitro by mesenchymal condensation., Proc. Natl. Acad. Sci. U. S. A. 111 (2014) 6940-5. doi:10.1073/pnas.1324050111.

[33] K. Mithoefer, T. Mcadams, R.J. Williams, P.C. Kreuz, B.R. Mandelbaum, Clinical efficacy of the microfracture technique for articular cartilage repair in the knee: An evidence-based systematic analysis, Am. J. Sports Med. 37 (2009) 2053-2063. doi:10.1177/0363546508328414.

[34] S. Wakitani, M. Nawata, K. Tensho, T. Okabe, H. Machida, H. Ohgushi, Repair of articular cartilage defects in the patello-femoral joint with autologous bone marrow mesenchymal cell transplantation: Three case reports involving nine defects in five knees, J. Tissue Eng. Regen. Med. 1 (2007) 74-79. doi:10.1002/term.8.

[35] H.S. McCarthy, S. Roberts, A histological comparison of the repair tissue formed when using either Chondrogide ${ }^{\circledR}$ or periosteum during autologous chondrocyte implantation, Osteoarthr. Cartil. 21 (2013) 2048-2057. doi:10.1016/j.joca.2013.10.004.

[36] A.M. Craft, J.S. Rockel, Y. Nartiss, R.A. Kandel, B.A. Alman, G.M. Keller, Generation of articular chondrocytes from human pluripotent stem cells, Nat. Biotechnol. 33 (2015) 638-645. doi:10.1038/nbt.3210.

[37] A. Yamashita, M. Morioka, Y. Yahara, M. Okada, T. Kobayashi, S. Kuriyama, S. Matsuda, N. Tsumaki, Generation of scaffoldless hyaline cartilaginous tissue from human iPSCs., Stem Cell Reports. 4 (2015) 404-18. doi:10.1016/j.stemcr.2015.01.016.

[38] A. Yamashita, Y. Tamamura, M. Morioka, P. Karagiannis, N. Shima, N. Tsumaki, Considerations in hiPSC-derived cartilage for articular cartilage repair, Inflamm. Regen. 38 (2018) 1-7. doi:10.1186/s41232-018-0075-8.

[39] J. Eyckmans, S.J. Roberts, J. Schrooten, F.P. Luyten, A clinically relevant model of 
osteoinduction: A process requiring calcium phosphate and BMP/Wnt signalling, J. Cell. Mol. Med. 14 (2010) 1845-1856. doi:10.1111/j.1582-4934.2009.00807.x.

[40] J. Leijten, L.S. Moreira Teixeira, J. Bolander, W. Ji, B. Vanspauwen, J. Lammertyn, J. Schrooten, F.P. Luyten, Bioinspired seeding of biomaterials using three dimensional microtissues induces chondrogenic stem cell differentiation and cartilage formation under growth factor free conditions, Sci. Rep. 6 (2016) 1-12. doi:10.1038/srep36011.

[41] L.F. Mendes, W.L. Tam, Y.C. Chai, L. Geris, F.P. Luyten, S.J. Roberts, Combinatorial Analysis of Growth Factors Reveals the Contribution of Bone Morphogenetic Proteins to Chondrogenic Differentiation of Human Periosteal Cells, Tissue Eng. Part C Methods. 22 (2016) 473-486. doi:10.1089/ten.tec.2015.0436.

[42] C.A. Schneider, W.S. Rasband, K.W. Eliceiri, NIH Image to ImageJ: 25 years of image analysis, Nat. Methods. 9 (2012) 671-675. doi:10.1038/nmeth.2089.

[43] T. Deckers, T. Lambrechts, S. Viazzi, G.N. Hall, I. Papantoniou, V. Bloemen, J.M. Aerts, High-throughput image-based monitoring of cell aggregation and microspheroid formation, PLoS One. (2018). doi:10.1371/journal.pone.0199092.

[44] K.J. Livak, T.D. Schmittgen, Analysis of relative gene expression data using real-time quantitative PCR and, Methods. 25 (2001) 402-408. doi:10.1006/meth.2001.1262.

[45] G. Kerckhofs, J. Sainz, M. Marechal, M. Wevers, T. Van de Putte, L. Geris, J. Schrooten, Contrast-Enhanced Nanofocus X-Ray Computed Tomography Allows Virtual ThreeDimensional Histopathology and Morphometric Analysis of Osteoarthritis in Small Animal Models, Cartilage. 5 (2013) 55-65. doi:10.1177/1947603513501175.

[46] W.A. Fernando, I. Papantoniou, L.F. Mendes, G. Nilsson Hall, K. Bosmans, W.L. Tam, L.M. Teixeira, M. Moos, L. Geris, F.P. Luyten, Limb derived cells as a paradigm for engineering self-assembling skeletal tissues, J. Tissue Eng. Regen. Med. (2017) 1-14. doi:10.1002/term.2498.

[47] A.C. Ruifrok, D.A. Johnston, Quantification of histochemical staining by color 
deconvolution., Anal. Quant. Cytol. Histol. 23 (2001) 291-299.

[48] J. Schindelin, I. Arganda-Carreras, E. Frise, V. Kaynig, M. Longair, T. Pietzsch, S. Preibisch, C. Rueden, S. Saalfeld, B. Schmid, J.Y. Tinevez, D.J. White, V. Hartenstein, K. Eliceiri, P. Tomancak, A. Cardona, Fiji: An open-source platform for biologicalimage analysis, Nat. Methods. (2012). doi:10.1038/nmeth.2019.

[49] S. Berg, D. Kutra, T. Kroeger, C.N. Straehle, B.X. Kausler, C. Haubold, M. Schiegg, J. Ales, T. Beier, M. Rudy, K. Eren, J.I. Cervantes, B. Xu, F. Beuttenmueller, A. Wolny, C. Zhang, U. Koethe, F.A. Hamprecht, A. Kreshuk, Ilastik: Interactive Machine Learning for (Bio)Image Analysis, Nat. Methods. 16 (2019) 1226-1232. doi:10.1038/s41592-019-0582-9.

[50] J.C.H. Leijten, J. Emons, C. Sticht, S. Van Gool, E. Decker, A. Uitterlinden, G. Rappold, A. Hofman, F. Rivadeneira, S. Scherjon, J.M. Wit, J. Van Meurs, C.A. Van Blitterswijk, M. Karperien, Gremlin 1, frizzled-related protein, and dkk-1 are key regulators of human articular cartilage homeostasis, Arthritis Rheum. $64 \quad$ (2012) 3302-3312. doi:10.1002/art.34535.

[51] M.S. Bergholt, J.P. St-Pierre, G.S. Offeddu, P.A. Parmar, M.B. Albro, J.L. Puetzer, M.L. Oyen, M.M. Stevens, Raman spectroscopy reveals new insights into the zonal organization of native and tissue-engineered articular cartilage, ACS Cent. Sci. 2 (2016) 885-895. doi:10.1021/acscentsci.6b00222.

[52] L.J. Power, C. Fasolato, A. Barbero, D.J. Wendt, A. Wixmerten, I. Martin, M.A. Asnaghi, Sensing tissue engineered cartilage quality with Raman spectroscopy and statistical learning for the development of advanced characterization assays, Biosens. Bioelectron. 166 (2020) 112467. doi:10.1016/j.bios.2020.112467.

[53] M.S. Bergholt, M.B. Albro, M.M. Stevens, Online quantitative monitoring of live cell engineered cartilage growth using diffuse fiber-optic Raman spectroscopy, Biomaterials. 140 (2017) 128-137. doi:10.1016/j.biomaterials.2017.06.015. 
[54] S. Brézillon, V. Untereiner, H.T. Mohamed, J. Hodin, A. Chatron-Colliet, F.X. Maquart, G.D. Sockalingum, Probing glycosaminoglycan spectral signatures in live cells and their conditioned media by Raman microspectroscopy, Analyst. 142 (2017) 1333-1341. doi:10.1039/c6an01951j.

[55] S. Gamsjaeger, R. Mendelsohn, A.L. Boskey, S. Gourion-Arsiquaud, K. Klaushofer, E.P. Paschalis, Vibrational spectroscopic imaging for the evaluation of matrix and mineral chemistry, Curr. Osteoporos. Rep. 12 (2014) 454-464. doi:10.1007/s11914-014-0238-8.

[56] J.J. Ng, Y. Wei, B. Zhou, J. Bernhard, S. Robinson, A. Burapachaisri, X.E. Guo, G. Vunjak-Novakovic, Recapitulation of physiological spatiotemporal signals promotes in vitro formation of phenotypically stable human articular cartilage., Proc. Natl. Acad. Sci. U. S. A. 114 (2017) 2556-2561. doi:10.1073/pnas.1611771114.

[57] M.A. Skylar-Scott, S.G.M. Uzel, L.L. Nam, J.H. Ahrens, R.L. Truby, S. Damaraju, J.A. Lewis, Biomanufacturing of organ-specific tissues with high cellular density and embedded vascular channels, Sci. Adv. 5 (2019) eaaw2459. doi:10.1126/sciadv.aaw2459.

[58] P. Occhetta, S. Pigeot, M. Rasponi, B. Dasen, A. Mehrkens, T. Ullrich, I. Kramer, S. Guth-Gundel, A. Barbero, I. Martin, Developmentally inspired programming of adult human mesenchymal stromal cells toward stable chondrogenesis, Proc. Natl. Acad. Sci. U. S. A. 115 (2018) 4625-4630. doi:10.1073/pnas.1720658115.

[59] L. Ling, X. Ren, X. Cao, A.B.M. Hassan, S. Mah, P. Sathiyanathan, R.A.A. Smith, C.L.L. Tan, M. Eio, R.M. Samsonraj, A.J. van Wijnen, M. Raghunath, V. Nurcombe, J.H. Hui, S.M. Cool, Enhancing the Efficacy of Stem Cell Therapy with Glycosaminoglycans., Stem Cell Reports. 14 (2019) 1-17. doi:10.1016/j.stemcr.2019.12.003.

[60] R. Castro-Viñuelas, C. Sanjurjo-Rodríguez, M. Piñeiro-Ramil, T. Hermida-Gómez, I.M. Fuentes-Boquete, F.J. de Toro-Santos, F.J. Blanco-García, S.M. Díaz-Prado, Induced pluripotent stem cells for cartilage repair: Current status and future perspectives, Eur. 
Cells Mater. 36 (2018) 96-109. doi:10.22203/eCM.v036a08.

[61] S. Diederichs, F.A.M. Klampfleuthner, B. Moradi, W. Richter, Chondral Differentiation of Induced Pluripotent Stem Cells Without Progression Into the Endochondral Pathway, Front. Cell Dev. Biol. 7 (2019) 270. doi:10.3389/fcell.2019.00270.

[62] Y. Li, L. Li, Z.N. Chen, G. Gao, R. Yao, W. Sun, Engineering-derived approaches for iPSC preparation, expansion, differentiation and applications, Biofabrication. 9 (2017) 032001. doi:10.1088/1758-5090/aa7e9a.

[63] M. Schnabel, S. Marlovits, G. Eckhoff, I. Fichtel, L. Gotzen, V. Vécsei, J. Schlegel, Dedifferentiation-associated changes in morphology and gene expression in primary human articular chondrocytes in cell culture, Osteoarthr. Cartil. 10 (2002) 62-70. doi:10.1053/joca.2001.0482.

[64] F.P. Luyten, F. Dell'Accio, C. De Bari, Skeletal tissue engineering: opportunities and challenges., Best Pract. Res. Clin. Rheumatol. $15 \quad$ (2001) 759-69. doi:10.1053/berh.2001.0192.

[65] N.P. Omelyanenko, P.A. Karalkin, E.A. Bulanova, E. V. Koudan, V.A. Parfenov, S.A. Rodionov, A.D. Knyazeva, V.A. Kasyanov, I.I. Babichenko, T.Z. Chkadua, Y.D. Khesuani, A.A. Gryadunova, V.A. Mironov, Extracellular Matrix Determines Biomechanical Properties of Chondrospheres during Their Maturation In Vitro, Cartilage. (2018). doi:10.1177/1947603518798890.

[66] S. Bhumiratana, R.E. Eton, S.R. Oungoulian, L.Q. Wan, G. a Ateshian, G. VunjakNovakovic, Large, stratified, and mechanically functional human cartilage grown in vitro by mesenchymal condensation., Proc. Natl. Acad. Sci. U. S. A. 111 (2014) 6940-5. doi:10.1073/pnas.1324050111.

[67] Z. Lin, Z. Li, E.N. Li, X. Li, C.J. Del Duke, H. Shen, T. Hao, B. O’Donnell, B.A. Bunnell, S.B. Goodman, P.G. Alexander, R.S. Tuan, H. Lin, Osteochondral Tissue Chip Derived From iPSCs: Modeling OA Pathologies and Testing Drugs, Front. Bioeng. Biotechnol. 
7 (2019) 1-16. doi:10.3389/fbioe.2019.00411.

[68] P.J. Roughley, J.S. Mort, The role of aggrecan in normal and osteoarthritic cartilage, J. Exp. Orthop. 1 (2014) 1-11. doi:10.1186/s40634-014-0008-7.

[69] S.R. Goldring, M.B. Goldring, Changes in the osteochondral unit during osteoarthritis: Structure, function and cartilage bone crosstalk, Nat. Rev. Rheumatol. 12 (2016) 632644. doi:10.1038/nrrheum.2016.148.

[70] C.D. Hoemann, C.H. Lafantaisie-Favreau, V. Lascau-Coman, G. Chen, J. GuzmánMorales, The cartilage-bone interface., J. Knee Surg. 25 (2012) 085-098. doi:10.1055/s0032-1319782.

[71] H. Da, S.J. Jia, G.L. Meng, J.H. Cheng, W. Zhou, Z. Xiong, Y.J. Mu, J. Liu, The Impact of Compact Layer in Biphasic Scaffold on Osteochondral Tissue Engineering, PLoS One. 8 (2013) e54838. doi:10.1371/journal.pone.0054838.

[72] W.D. Lee, M.B. Hurtig, R.M. Pilliar, W.L. Stanford, R.A. Kandel, Engineering of hyaline cartilage with a calcified zone using bone marrow stromal cells, Osteoarthr. Cartil. 23 (2015) 1307-1315. doi:10.1016/j.joca.2015.04.010.

[73] L.F. Mellor, R.C. Nordberg, P. Huebner, M. Mohiti-Asli, M.A. Taylor, W. Efird, J.T. Oxford, J.T. Spang, R.A. Shirwaiker, E.G. Loboa, Investigation of multiphasic 3Dbioplotted scaffolds for site-specific chondrogenic and osteogenic differentiation of human adipose-derived stem cells for osteochondral tissue engineering applications, J. Biomed. Mater. Res. - Part B Appl. Biomater. 108 (2020) 2017-2030. doi:10.1002/jbm.b.34542.

[74] I. Kiviranta, J. Jurvelin, M. Tammi, A. - M SääMäunen, H.J. Helminen, Weight bearing controls glycosaminoglycan concentration and articualr cartilage thickness in the knee joints of young beagle dogs, Arthritis Rheum. 30 (1987) 801-809. doi:10.1002/art.1780300710.

[75] J. Ng, Y. Wei, B. Zhou, S. Bhumiratana, A. Burapachaisri, E. Guo, G. Vunjak- 
Novakovic, Ectopic implantation of juvenile osteochondral tissues recapitulates endochondral ossification, J. Tissue Eng. Regen. Med. 12 (2018) 468-478. doi:10.1002/term.2500.

[76] H. Koike, K. Iwasawa, R. Ouchi, M. Maezawa, K. Giesbrecht, N. Saiki, A. Ferguson, M. Kimura, W.L. Thompson, J.M. Wells, A.M. Zorn, T. Takebe, Modelling human hepatobiliary-pancreatic organogenesis from the foregut-midgut boundary, Nature. 574 (2019) 112-116. doi:10.1038/s41586-019-1598-0.

[77] P. Samal, C. van Blitterswijk, R. Truckenmüller, S. Giselbrecht, Grow with the Flow: When Morphogenesis Meets Microfluidics, Adv. Mater. 31 (2019). doi:10.1002/adma.201805764.

[78] G.M. De Peppo, I. Marcos-Campos, D.J. Kahler, D. Alsalman, L. Shang, G. VunjakNovakovic, D. Marolt, Engineering bone tissue substitutes from human induced pluripotent stem cells, Proc. Natl. Acad. Sci. U. S. A. 110 (2013) 8680-8685. doi:10.1073/pnas.1301190110.

[79] C. Jacobsen, A.M. Craft, Retinoic-acid-induced osteogenesis of hiPSCs, Nat. Biomed. Eng. 3 (2019) 504-506. doi:10.1038/s41551-019-0422-3.

[80] B. Ayan, D.N. Heo, Z. Zhang, M. Dey, A. Povilianskas, C. Drapaca, I.T. Ozbolat, Aspiration-assisted bioprinting for precise positioning of biologics, Sci. Adv. 6 (2020) 1-17. doi:10.1126/sciadv.aaw5111.

[81] A.C. Daly, D.J. Kelly, Biofabrication of spatially organised tissues by directing the growth of cellular spheroids within 3D printed polymeric microchambers, Biomaterials. 197 (2019) 194-206. doi:10.1016/j.biomaterials.2018.12.028.

[82] N. V. Mekhileri, K.S. Lim, G.C.J. Brown, I. Mutreja, B.S. Schon, G.J. Hooper, T.B.F. Woodfield, Automated 3D bioassembly of micro-tissues for biofabrication of hybrid tissue engineered constructs, Biofabrication. 10 (2018) 024103. doi:10.1088/17585090/aa9ef1. 
[83] A. Di Luca, I. Lorenzo-Moldero, C. Mota, A. Lepedda, D. Auhl, C. Van Blitterswijk, L. Moroni, Tuning Cell Differentiation into a 3D Scaffold Presenting a Pore Shape Gradient for Osteochondral Regeneration, Adv. Healthc. Mater. 5 (2016) 1753-1763. doi:10.1002/adhm.201600083. 


\section{Figures - Figure 1}

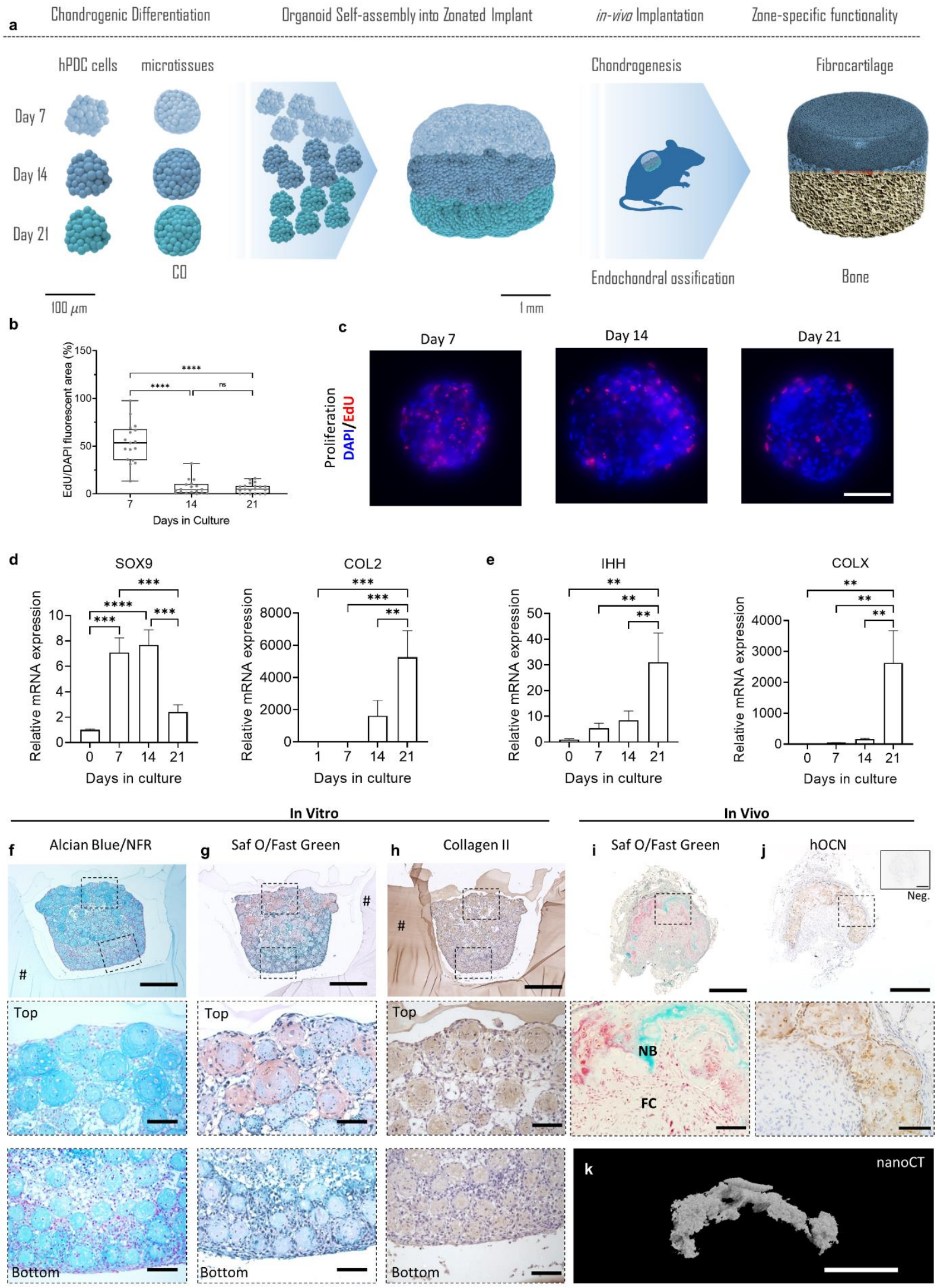


Figure 2
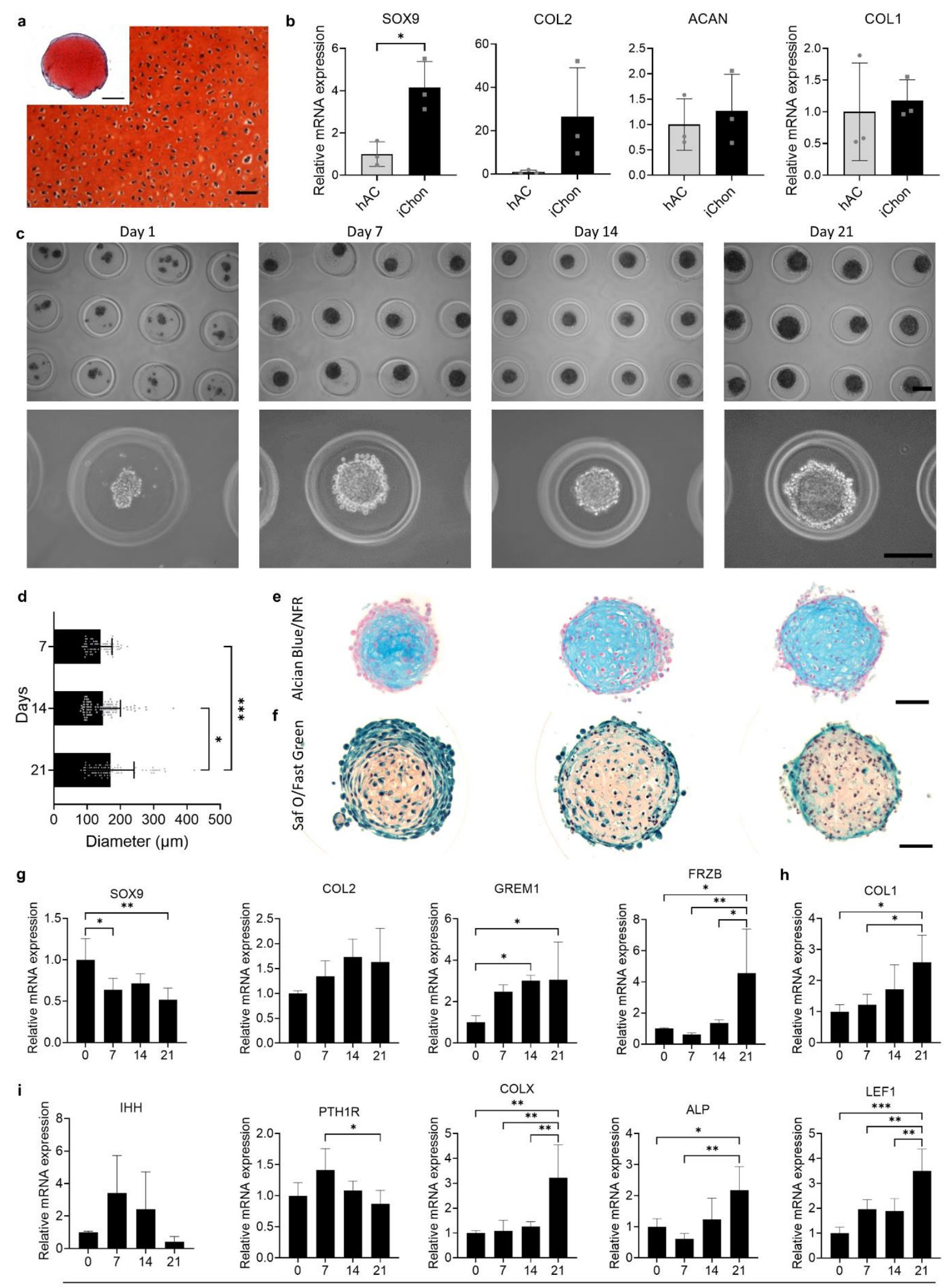

Days in culture 


\section{Figure 3}
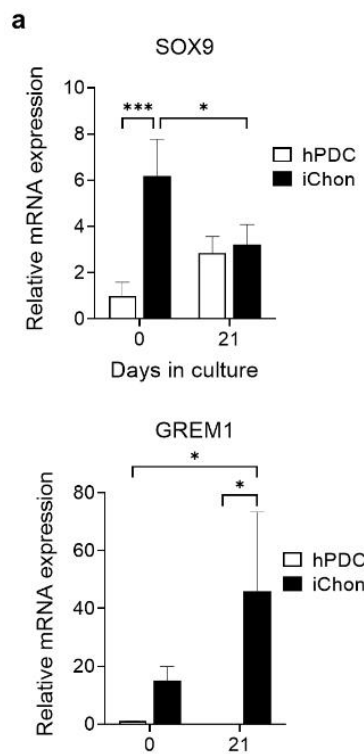

Days in culture

c

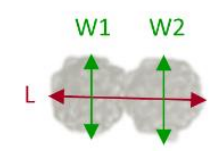

$A R=\frac{L}{(W 1+W 2) / 2}$
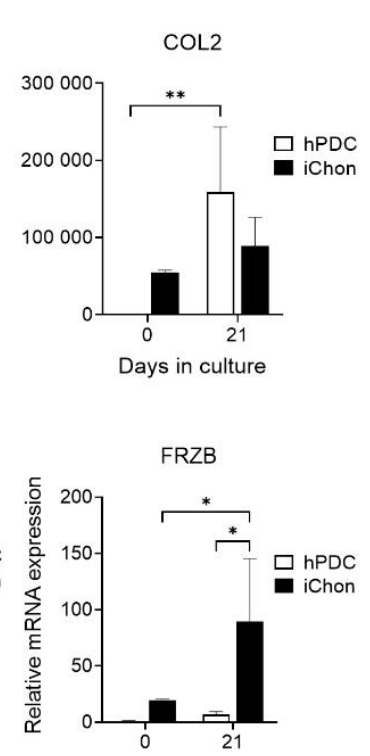

Days in culture
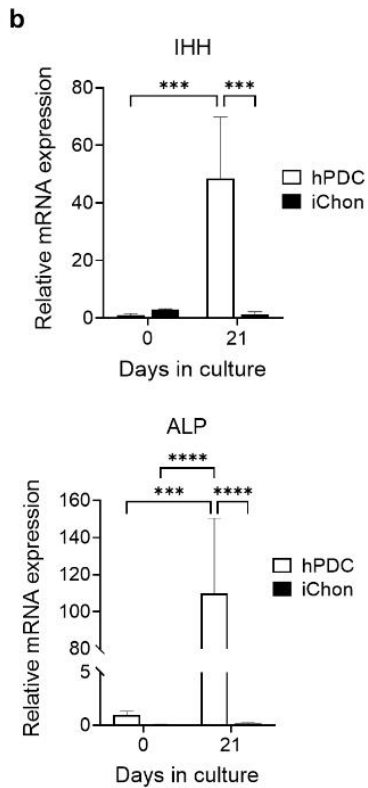

Days in culture

e $\cos (\mathrm{hPDC})$ iCMTs (iChon) f

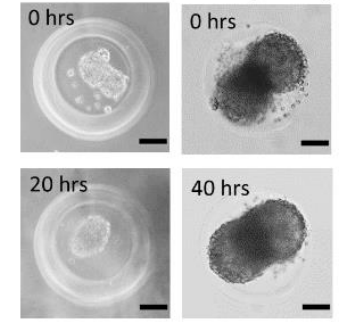

COLX

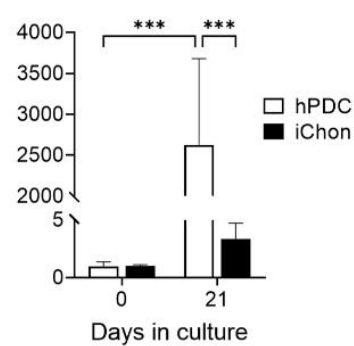

PTH1R

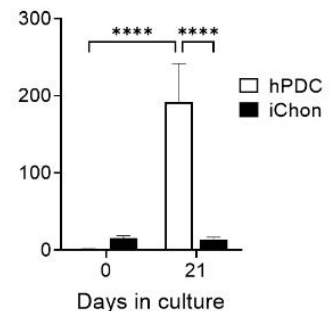

Days in culture d Dual building block fusion

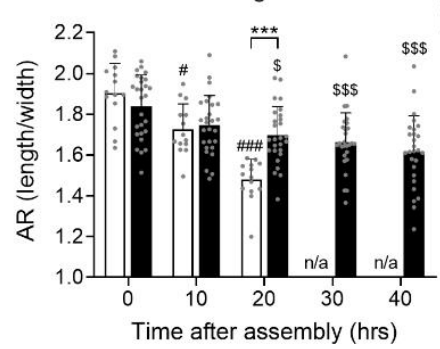
iCMT

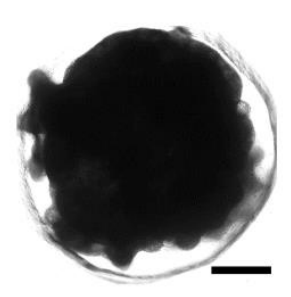




\section{Figure 4}
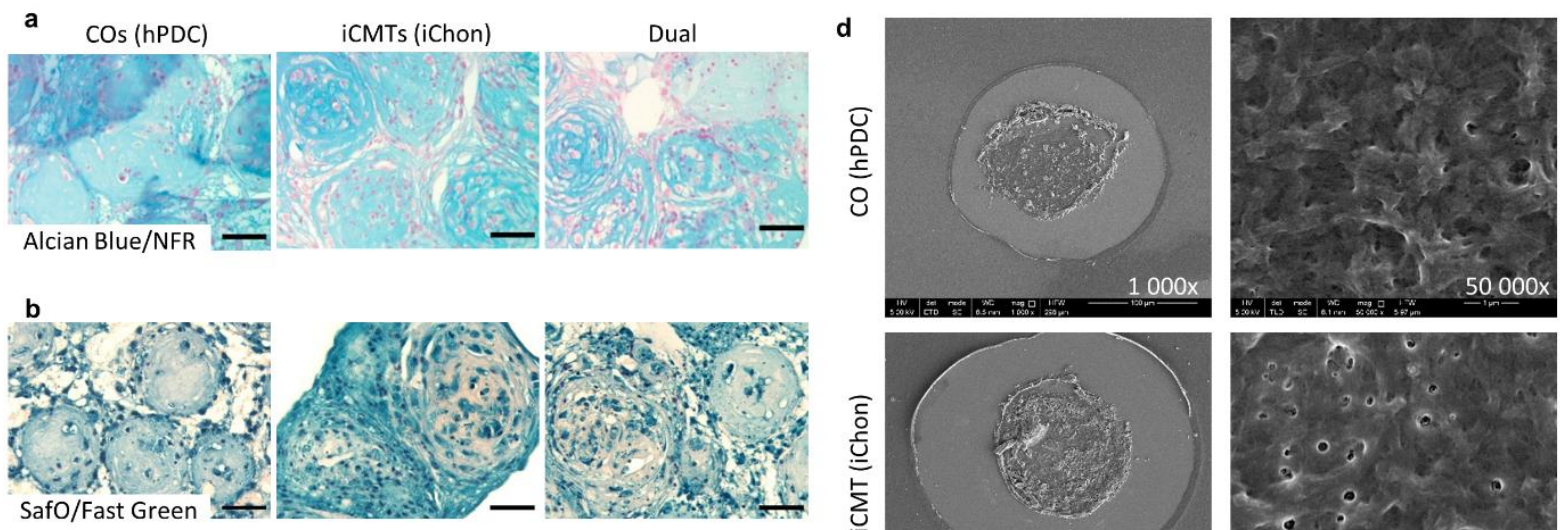

c
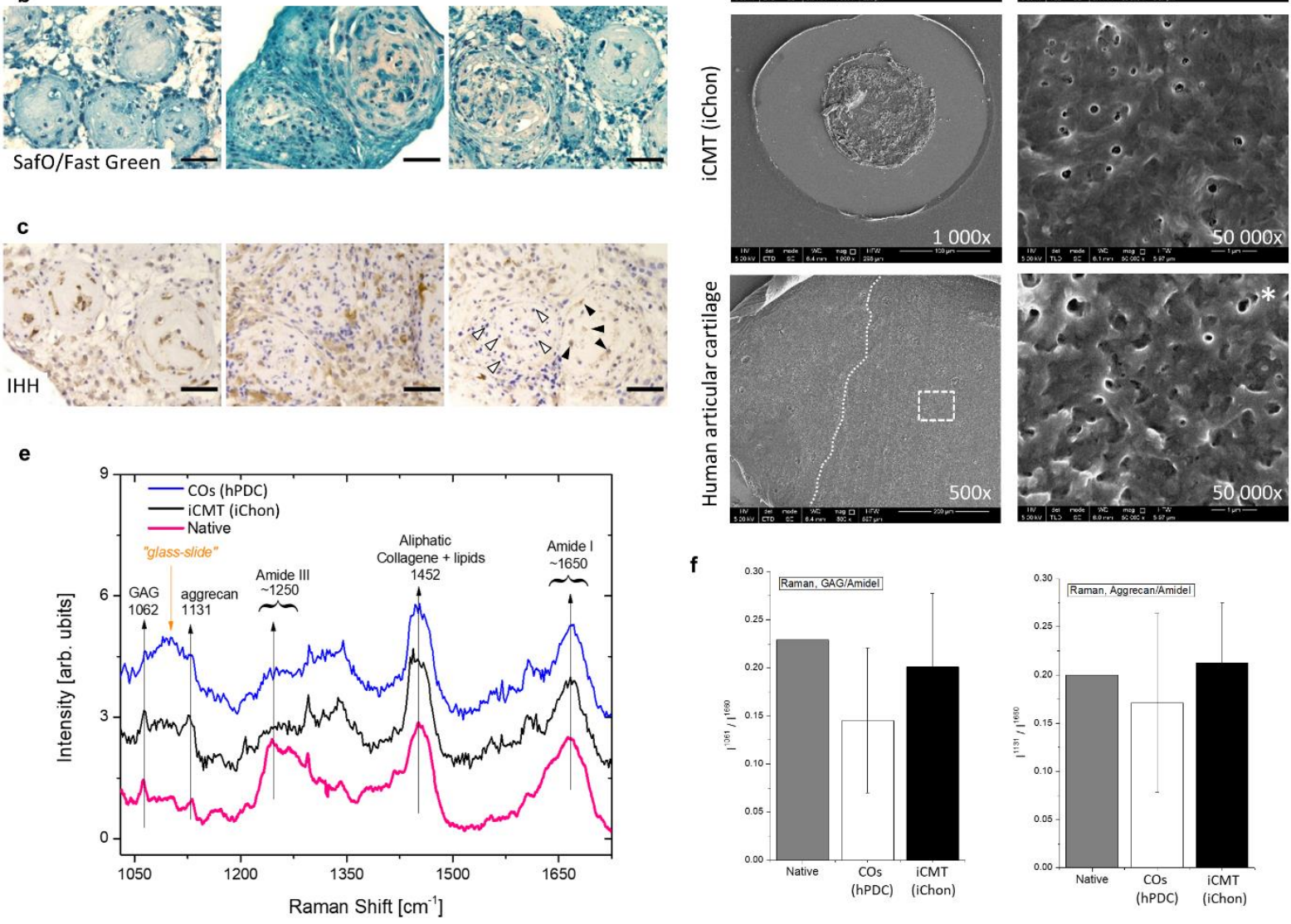


\section{Figure 5}
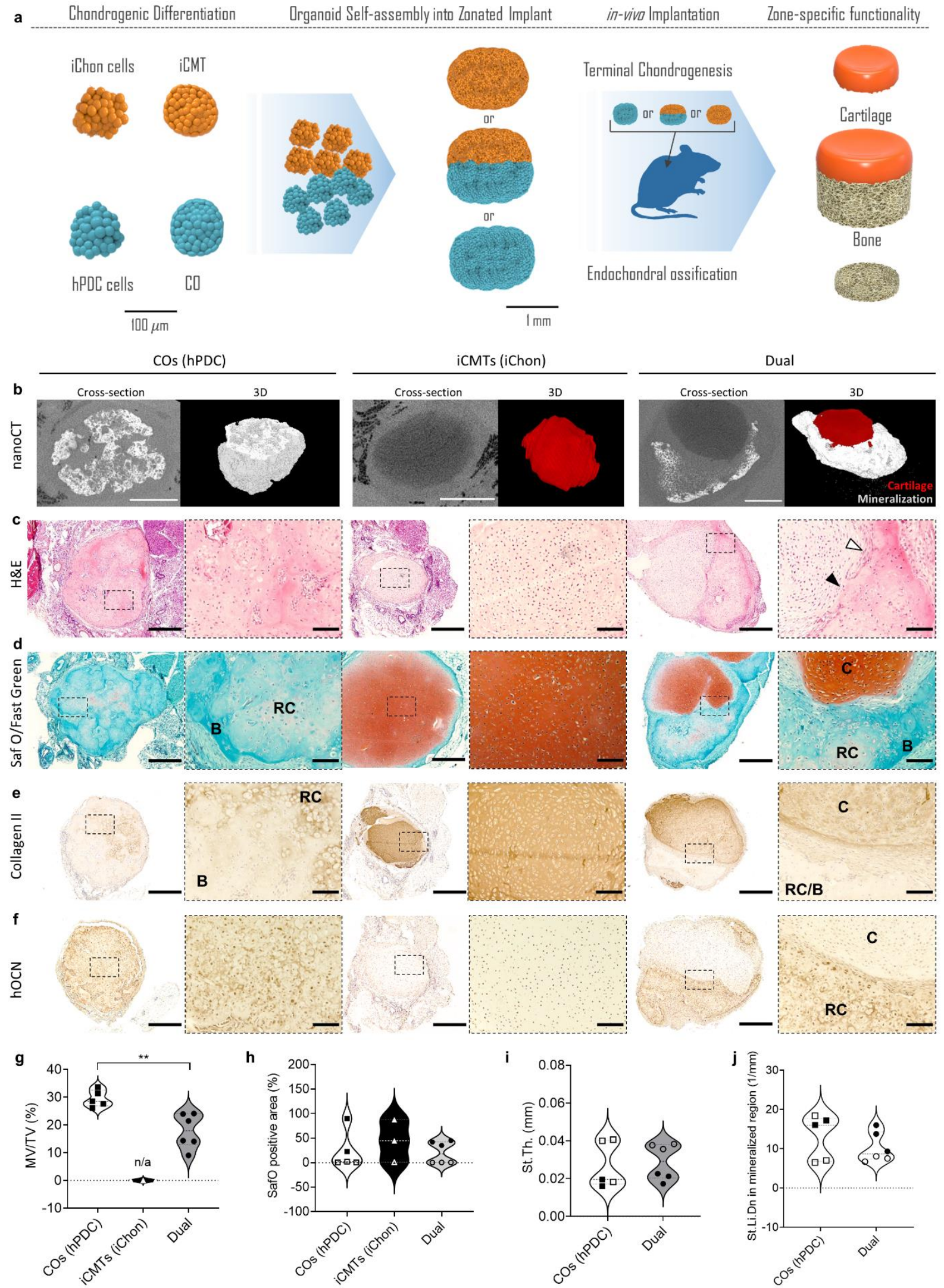


\section{Figure Captions}

Figure 1. Hierarchical micromodule implant derived from adult hPDCs. a) Schematic overview of building block assembly into tri-layered constructs. b) Semi-quantification of proliferating EdU positive cells (box plot min to max where each point represents one microspheroid; oneway ANOVA followed by Tukey's post-hoc test) and c) representative images. d) Gene expression analysis on the different building block populations before assembly of chondrogenic and e) hypertrophic markers (graphs show mean \pm SD of three independent samples; one-way ANOVA followed by Tukey's post-hoc test). f) In vitro constructs stained with Alcian blue/Nuclear Fast Red (NFR), g) Safranin O/Fast Green and h) collagen type II immunostaining. Samples four weeks after implantation stained with i) Safranin O/Fast Green and j) human osteocalcin (hOCN). k) 3D visualization of mineralized tissue imaged with nanoCT; 3 constructs were implanted in 3 different mice. \#: agarose, FC: fibrocartilage, NB: new bone. Scale bars represent c: $50 \mu \mathrm{m}, \mathrm{f}$-j: top-images represent $500 \mu \mathrm{m}$, zoom-in images $100 \mu \mathrm{m}$ and $\mathrm{k}: 500 \mu \mathrm{m}$.

Figure 2. Formation of cartilage microtissues from iPSC derived chondrocytes (iChon). a) Safranin O /Fast Green staining of mature chondrogenic nodules derived from iPSCs. b) Gene expression comparison of cells isolated from iChon nodules and hAC (mean $\pm \mathrm{SD}$; each data point represents one hAC donor/independent iPSC experiment; unpaired t test). c) Bright field images of building blocks created from iChon in non-adherent microwells. d) Projection area of iChon building blocks over time (mean $\pm \mathrm{SD}$; each data point represents one individual building block, data pooled from 3 independent iPSC experiments; one-way ANOVA followed by Tukey's post-hoc test). Histological sections of cartilaginous building blocks stained with e) Alcian blue/NFR and f) Saf O/Fast Green. Gene expression analysis of iChon building blocks over time of genes related to g) articular cartilage, h) fibrocartilage and i) chondrocyte hypertrophy (mean \pm SD of four independent samples; one-way ANOVA followed by Tukey's post-hoc test). Scale bars represent a: $50 \mu \mathrm{m}$ (zoom-in) and $500 \mu \mathrm{m}$ (insert), c: $200 \mu \mathrm{m}$ and ef: $50 \mu \mathrm{m}$.

Figure 3. Creation of integrated osteochondral tissues through assembly of hPDC- and iChonderived building blocks. a) Chondrogenic and b) hypertrophic gene expression analysis of hPDC- and iChon derived building blocks at day 0 and 21 (mean \pm SD of three (hPDC) or four (iChon) independent samples; two-way ANOVA followed by Tukey's post-hoc test). c) Equation for the aspect ratio (AR). d) Aspect ratio (AR) quantification for dual building block fusion over time for callus organoids (COs) and cartilage microtissues (iCMTs) separately (mean $\pm \mathrm{SD}$, each data point represents one dual fusion; two-way ANOVA followed by Tukey's post-hoc test; \#: compared to COs 0hrs; \$: compared to iCMTs 0hrs). e) Representative images of fusing building blocks. f) Bright field image of a fused construct in the constructed agarose macro-well. AR: aspect ratio, L: length, W: width, COs: callus organoids, iCMTs: cartilage microtissues. Scale bars represent e: $100 \mu \mathrm{m}$, f: $500 \mu \mathrm{m}$. 
Figure 4. Characterization of the extracellular matrix in building blocks. Histological sections of in vitro constructs stained with a) Alcian blue/NFR, b) Saf O/Fast Green and c) indian hedgehog ( $\mathrm{IHH}$ ) immunostaining (white arrows: cells negative for IHH; black arrows: cells positive for IHH). d) SEM images of CO, iCMT building blocks and human articular cartilage (white dotted line: tidemark, *: articular cartilage). e) Typical Raman spectra from native articular cartilage, COs and iCMTs. f) Bar charts indicating the relative intensity of the 1061 (GAG) and $1131 \mathrm{~cm}^{-1}$ (aggrecan) peaks with respect to the aliphatic $\sim 1650 \mathrm{~cm}^{-1}$ band (mean \pm SD). COs: callus organoids, iCMTs: cartilage microtissues, IHH: Indian hedgehog. Scale bars represent a-c: $50 \mu \mathrm{m}$.

Figure 5. In vivo functionality of integrated dual constructs after 4 weeks implantation. a) Schematic illustration of building block assembly into zonal constructs. b) Cross-section and 3D rendering of nanoCT demonstrating mineralized (white; threshold defined by automatic Otsu segmentation) and cartilaginous tissue (dark grey in cross-section and red in 3D rendering). Histological sections of samples after 4 weeks subcutaneous implantation stained with c) H\&E (arrows: zone interface), d) Safranin O/Fast Green (Figure S3d-e, Supporting Information), e) collagen type II and f) human osteocalcin (hOCN) immunostaining. g) Quantification of mineralized tissue normalized to total tissue volume (\% MV/TV). h) Quantification of Saf O stained regions (\%). i) Structure Thickness (St. Th.) and j) Structure Linear Density (St.Li.Dn) of the mineralized structure in the bony region calculated from nanoCT images. Graphs show violin plots with each data point representing one explant. 5 of 5 COs, 3 of 4 iCMTs and 6 of 6 Dual constructs were recuperated in total from two separate experiments; two samples per mouse were implanted ( 8 mice in total). B: bone, RC: remodeling cartilage, $\mathrm{C}$ : cartilage, COs: callus organoids, iCMTs: cartilage microtissues. Scale bars represent $500 \mu \mathrm{m}$ and zoom-ins (dashed border) $100 \mu \mathrm{m}$. 


\section{Supporting Information}

Patterned, organoid-based cartilaginous implants exhibit zone specific functionality forming osteochondral-like tissues in vivo

Gabriella Nilsson Hall ${ }^{1,2}$, Wai Long Tam², Konstantinos S. Andrikopoulos ${ }^{3,4}$, Leire Casas-

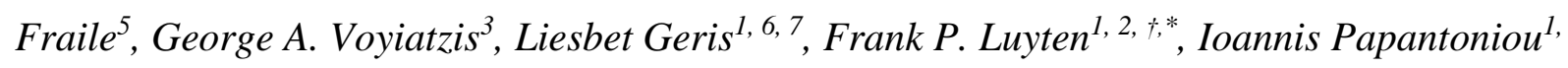
$2,3, *, *$

${ }^{1}$ Prometheus Division of Skeletal Tissue Engineering, KU Leuven, O\&N1, Herestraat 49, PB 813, 3000 Leuven, Belgium

${ }^{2}$ Skeletal Biology and Engineering Research Center, Department of Development and Regeneration, KU Leuven, O\&N1, Herestraat 49, PB 813, 3000 Leuven, Belgium

${ }^{3}$ Institute of Chemical Engineering Sciences, Foundation for Research and Technology-Hellas, Stadiou 26504, Platani, Patras, Greece

${ }^{4}$ Department of Physics, University of Patras, GR-265 00 Rio-Patras, Greece

${ }^{5}$ Laboratory of Tissue Homeostasis and Disease, Skeletal Biology and Engineering Research Center, Department of Development and Regeneration, KU Leuven, O\&N1, Herestraat 49, PB 813, Leuven 3000, Belgium

${ }^{6}$ GIGA in silico medicine, Université de Liège, Avenue de l'Hôpital 11 - BAT 34, 4000 Liège 1, Belgium

${ }^{7}$ Biomechanics Section, KU Leuven, Celestijnenlaan 300C, PB 2419, 3001 Leuven, Belgium

${ }^{\dagger}$ Co-senior authors.

${ }^{*}$ Corresponding Authors: Skeletal Biology and Engineering Research Center; Onderwijs en Navorsing 8th floor, bus 813; B-3000 Leuven, Belgium

Tel: +3216346138

Fax: +32 16346200

Email: ioannis.papantoniou@kuleuven.be

Email: frank.luyten@kuleuven.be 


\section{Supplementary Figures}
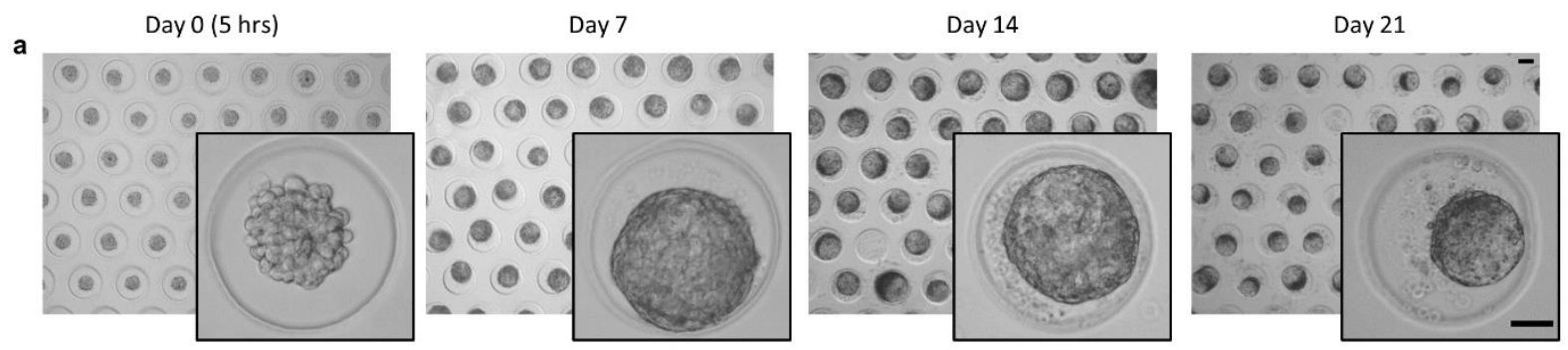

b

Alcian Blue/NFR
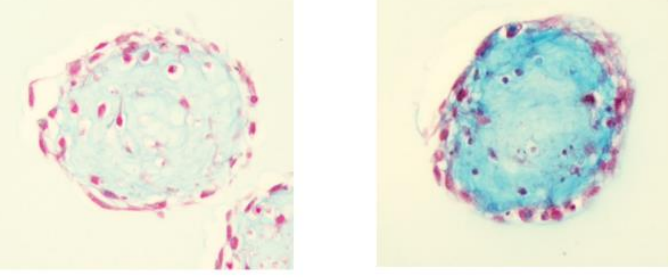

c

Saf $\mathrm{O} /$ Fast green
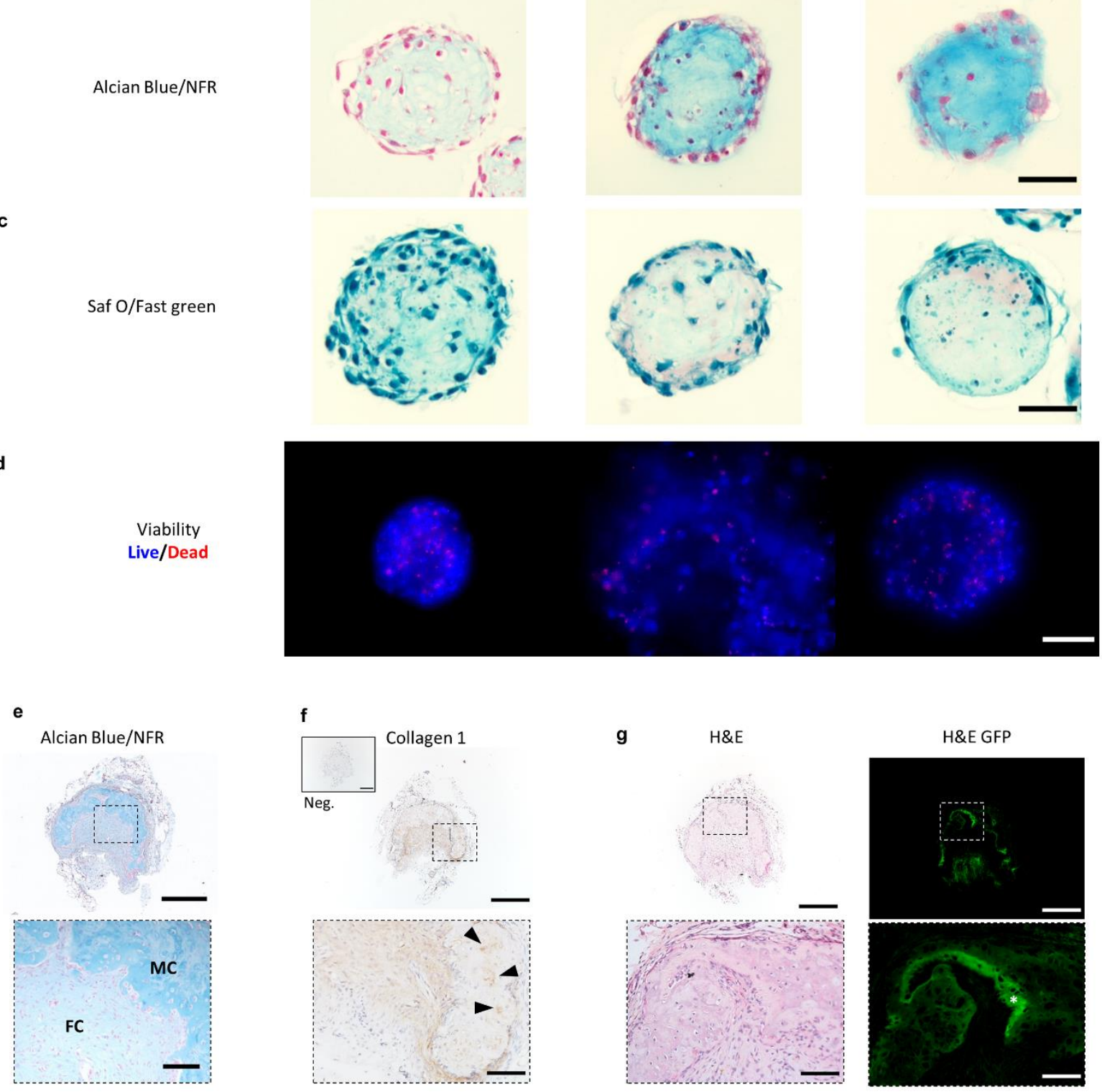

Figure S1. Formation of cartilaginous and prehypertrophic building blocks from adult hPDCs.

a) Bright field images of hPDC microspheroids over time. b) Alcian blue/NFR and c) Safranin $\mathrm{O}$ (Saf O) staining of building block sections at day 7, 14 and 21. d) Viability staining of hPDC microspheroids over time. Hierarchical constructs after four weeks implantation stained with e) Alcian Blue/NFR, f) collagen type I (insert: negative control without primary antibody), and 
H\&E visualized with bright field and GFP fluorescence. Scale bars represent a-d: $50 \mu \mathrm{m}$, e-g: top-images $500 \mu \mathrm{m}$ and zoom-in images $100 \mu \mathrm{m}$. 

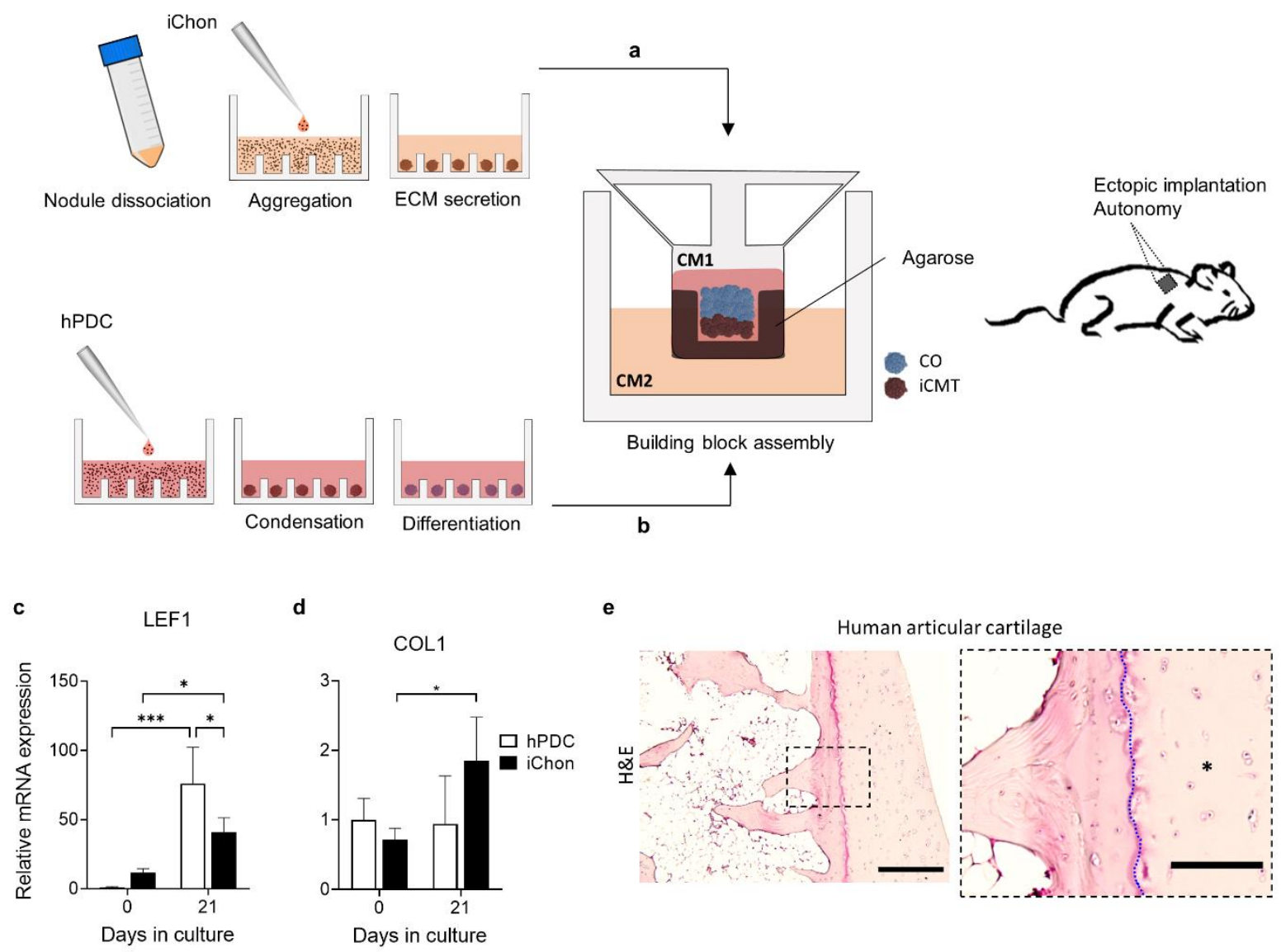

Figure S2. Schematic image of building block assembly into dual constructs. a) iChon were isolated, cultured as microspheroids into iCMTs and assembled into an agarose microwell (2 mm diameter). b) hPDCs cultured as microspheroids into COs were added after $24 \mathrm{hrs}$ for bilayered assembly. Bilayered constructs were implanted subcutaneously in immune compromised mice. c) $L E F 1$ and d) COL1 gene expression analysis of hPDC- and iChon derived building blocks at day 0 and 21 (mean \pm SD of three (hPDC) or four (iChon) independent samples; two-way ANOVA followed by Tukey's post-hoc test). e) H\&E staining of human osteochondral tissue, *: articular cartilage, dotted line: tide mark. hPDC: human periosteum-derived cells, iChon: iPSC-derived chondroprogenitors, CM: chondrogenic medium, COs: callus organoids, iCMTs: cartilage microtissues. Scale bars e) represent left: 500 $\mu \mathrm{m}$ and right: $200 \mu \mathrm{m}$. 
a

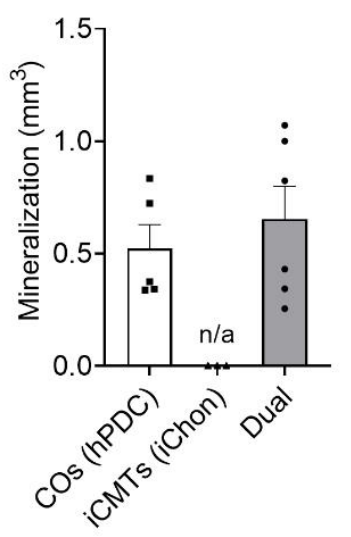

b

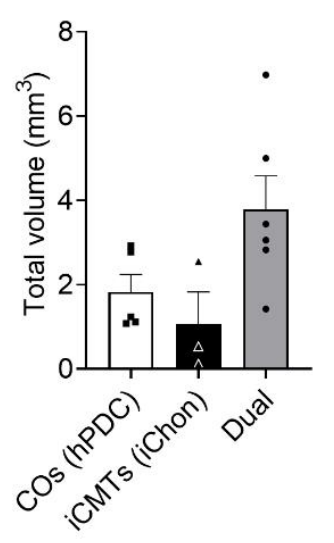

C

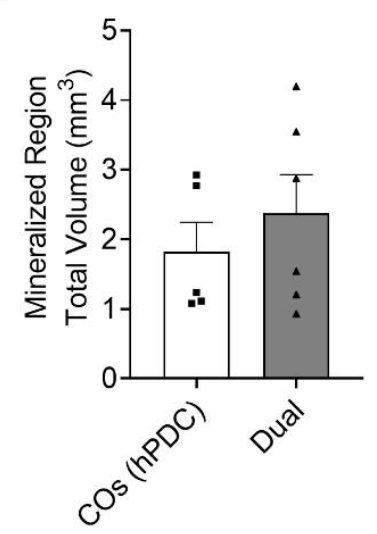

d

Dual integration - nanoCT

Dual integration - Saf O/Fast Green
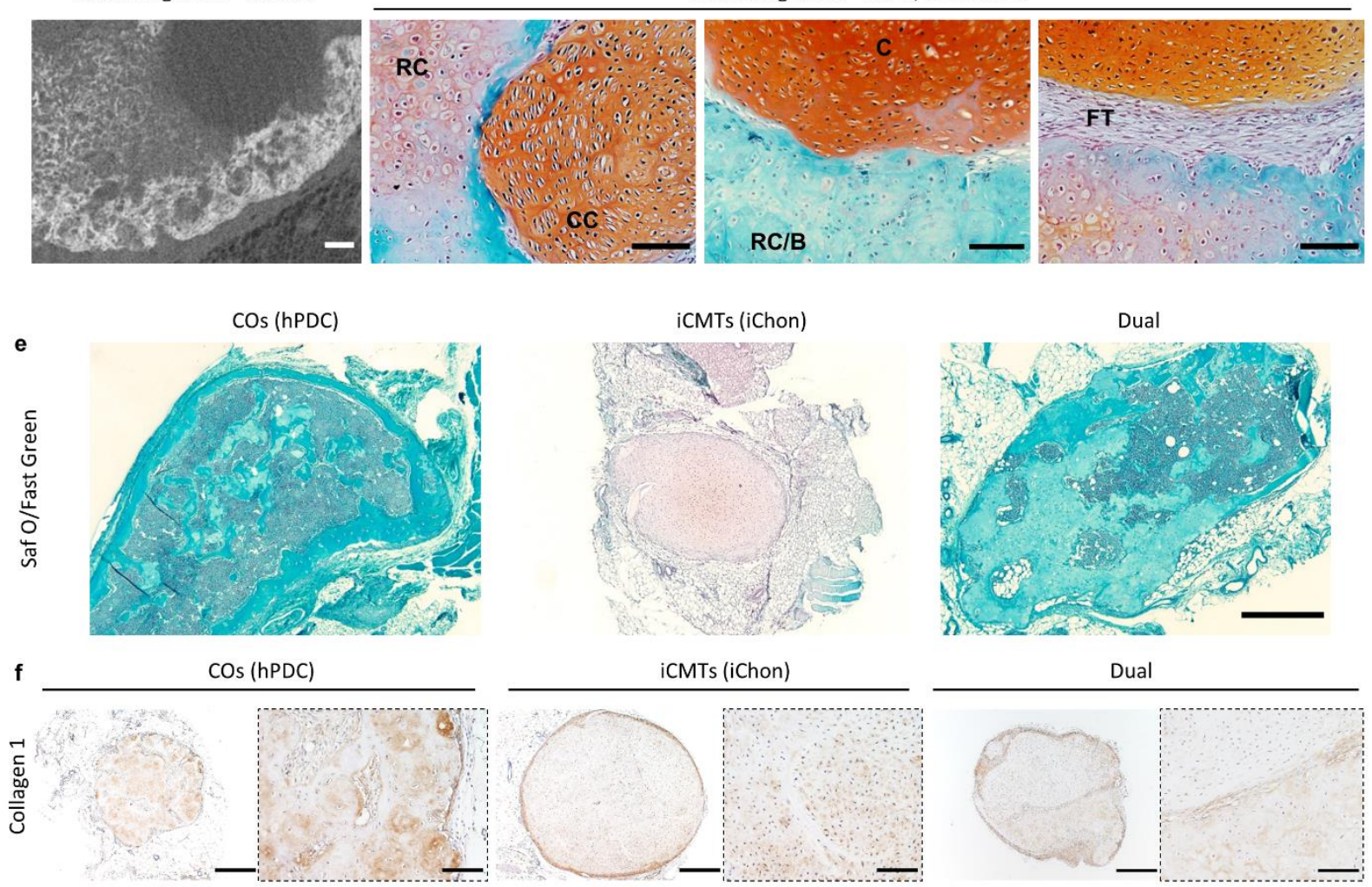

Dual

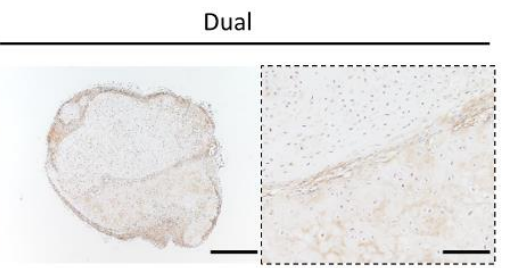

Figure S3. NanoCT analysis after 4 weeks implantation to define a) total mineralized volume b) total explant volume, and c) total volume of mineralized region (without hexabrix stained region). Graphs show mean \pm SEM with explants pooled from two individual experiments (COs: 5, iCMTs: 3, Dual: 6 samples); each data point represents one sample; one-way ANOVA followed by Tukey's post-hoc test; two samples per mouse were implanted ( 8 mice in total). d) nanoCT section and Saf O/Fast Green staining of three types of dual integration detected. e) Saf O/Fast green staining of alternative in vivo outcome. f) Collagen type 1 immunostaining of explants. COs forming full ossicle (2/5), iCMTs with weak Saf O staining (2/3) and Dual constructs without Saf O positive areas (3/6). RC: remodeling cartilage, CC: columnar chondrocytes, B: bone, FT: fibrous tissue, iChon: iPSC-derived chondroprogenitors, COs: 
callus organoids, iCMTs: cartilage microtissues. Scale bars represent d: $100 \mu \mathrm{m}$, e: $500 \mu \mathrm{m}, \mathrm{f}$ : $500 \mu \mathrm{m}$ and zoom-ins (dashed border) $100 \mu \mathrm{m}$. 